\title{
Welcome to CLEO:2016!
}

We welcome you to CLEO:2016. CLEO continues to be the world's premier international forum for scientific and technical optics, uniting the fields of lasers and opto-electronics by bringing together all aspects of laser technology, from basic research to industry applications. Within the scope of a single conference, CLEO provides a forum where attendees can explore new scientific ideas, engineering concepts, and emerging applications in fields such as biophotonics, optical communications, and novel light sources. While the quality of work presented remains assured by CLEO's world-renowned technical program, the conference continues to evolve with new features to enhance your experience.

\section{CLEO:2016 offers high quality content in five core event elements:}

CLEO Fundamental Science: the premier venue for discussion of basic research in optical and laser physics and related fields. Topics include optics, modern spectroscopy, optical materials, quantum information science, nanophotonics, plasmonics, and metamaterials.

CLEO Science \& Innovations: world-leading scientific research and innovation in lasers, optical materials, and photonic devices. Topics include laser processing of materials, terahertz technologies, ultrafast optics, biophotonics, nanophotonics, metrology, sensing, and energy-efficient "green" photonics.

CLEO Applications \& Technology: exploration of the transition of fundamental research into emerging applications and products. The scope spans innovative laser and EO components and systems and applications. This includes: biomedical devices for diagnostics and therapeutics, high power laser systems for industry and defense, environmental sensing, and energy conservation.

CLEO Market Focus: emphasis on commercial developments in the optics, photonics, and laser industry. The sessions will discuss how the power of photonics is expanding to be used by nontraditional optics \& photonics companies to leverage manufacturing and production accelerating products to markets. A Technology Transfer showcase will feature the latest research ready to be transitioned for commercial implementation.

CLEO Expo: exhibition of businesses who provide leading-edge products and services to CLEO attendees. The Expo will host more than 250 participating companies featuring a wide range of photonics innovations, products and services; it is expected to attract more than 4,400 attendees including researchers, engineers, and leaders from top research institutions and major businesses who represent the fastest growing markets in optics and photonics.

This year the conference features the largest collection of contributed papers, invited speakers and tutorials ever featured at CLEO. We are excited to offer more than 1,480 oral presentations, 440 poster presentations, as well as 169 invited talks by some of the leading researchers in our international community representing 48 countries. The program also includes 25 tutorials - hour-long in-depth presentations by experts - along with a comprehensive program of 17 short courses; which provide half day immersion in selected topic areas. The CLEO technical program committee ensures high technical quality in all presentations through a rigorous peer review system that is comprises of over 300 scientists serving on 25 technical committees.

This year the technical Program Chairs selected 8 special symposia that were submitted during an open call for submissions that consisted of timely, cutting-edge topics and/or new material in rapidly advancing areas. In addition, Applications and Technology Topical Reviews have been continued to add to emphasize in significant recent advances in the application of photonic technologies to address current real world problems.

This year's CLEO features 5 exceptional plenary speakers. On Monday we will hear from David Reitze the Executive Director of LIGO on LIGO's recent detection of gravitational waves. Tuesday afternoon will feature Masataka Nakazawa and Jelena Vuckovic. Nakazawa will describe recent developments toward a hardware paradigm shift required to overcome the capacity limitation imposed by the optical communication infrastructure, while Vuckovic will discuss Quantum nanophotonics. On Wednesday evening, we will be joined by Eicke Weber and Ferenc Krausz. Weber present multijunction solar cell structures of record performance and lay out materials challenges and opportunities for next generation industry-scale solar harvesting, and Krausz will conclude the program with a talk on revolutionary developments in ultrashort optical pulse technologies which brought about the era of physics on attosecond timescales.

We thank technical Program Co-chairs; Peter E. Andersen and Dirk H. Sutter in Applications and Technology; Zhigang Chen and Jeffrey H. Shapiro in Fundamental Science, Nathan R. Newbury and Jessie Rosenberg in Science and Innovations for coordinating the work of our subcommittees to compile the largest CLEO program. We also thank Sasan Fathpour and Ben Eggleton, Short Course Co-chairs, and all of the program committee members whose leadership, dedication, and hard work are critical to maintaining the high quality of the meeting. Additionally, we would like to thank the APS Division of Laser Science, the IEEE Photonics Society, The Optical Society (OSA), and the exhibitors for their support and contributions to the meeting. Finally, we thank the OSA staff for their professional assistance and dedication in organizing this event.

We welcome you to the conference and thank you for your participation.

\section{General Co-Chairs}

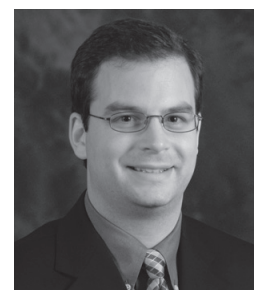

Seth Bank

Univ. of Texas at Austin, USA

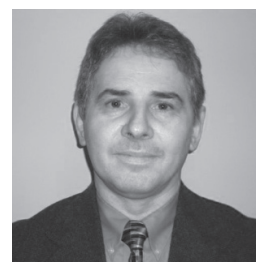

Nicusor Iftimia

Physical Sciences Inc., USA

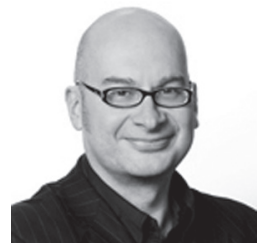

Roberto Morandotti INRS, Canada

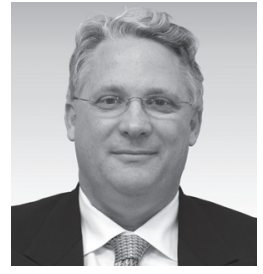

William Munro

NTT Basic Research Laboratories, Japan

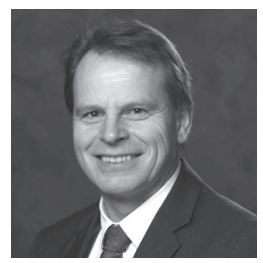

Christian Wetzel

Rensselaer Polytechnic Inst., USA

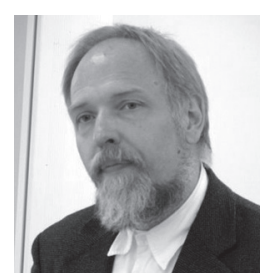

Valdas Pasiskevicius Royal Inst. of Technology $(K T H)$, Sweden 


\section{Plenary Sessions and Award Ceremony}

\section{Plenary Session I}

Monday, 6 June, 18:15-18:45

Grand Ballroom

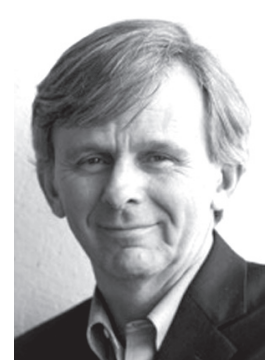

Colliding Black Holes \& Convulsions in Space-time: The First Observation of Gravitational Waves by LIGO

David Reitze, LIGO Laboratory, California Institute of Technology, USA

David Reitze holds joint positions as the Executive Director of the LIGO (Laser Interferometer Gravitational-wave Observatory) Lab at the California Institute of Technology and a Professor of Physics at the University of Florida. He received a Ph.D. in Physics from the University of Texas at Austin in ultrafast laser spectroscopy in 1990 and has worked extensively in the area of experimental gravitational-wave detection for 20 years. He has authored or co-authored over 250 peer-reviewed publications, and is a Fellow of the American Physical Society and The Optical Society.

\section{Plenary Session II}

Tuesday, 7 June, 10:30-11:30

Grand Ballroom

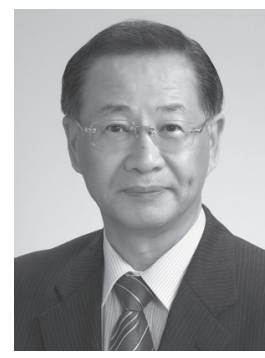

"Multi" is Everywhere

Masataka Nakazawa, Research Institute of Electrical Communication, Tohoku University, Japan

After receiving his Ph.D. from the Tokyo Institute of Technology in 1980, he joined NTT laboratory. He was a visiting scientist at MIT from 1984-1985. In 2001, he was appointed professor at Tohoku University, where he was the director of the Research Institute of Electrical Communication (RIEC). He engaged in research on erbium-doped fiber amplifiers (EDFA), optical solitons, ultrahigh-speed optical transmission, digital coherent transmission, and ultrashort pulse lasers. Among them, regeneratively and harmonically mode-locked fiber laser in the $10-40 \mathrm{GHz}$ region played an important role in high-speed transmission. Recently his research is focusing on digital coherent transmission with Nyquist pulses. He achieved 2048 QAM multi-level coherent transmission, which enabled a spectral efficiency of higher than $15 \mathrm{bit} / \mathrm{s} / \mathrm{Hz}$. He has published 460 papers and presented 300 international conference presentations. He has received many awards including an IEEE Quantum Electronics Award in 2010 and the Charles Hard Townes Award in 2014. He was the president of Electronics Society of the IEICE and a Board member of The Optical Society, and is now a Board member of the IEEE Photonics Society and the vice president of the IEICE. Dr. Nakazawa is a Fellow of the OSA, IEEE, IEICE, and JSAP.

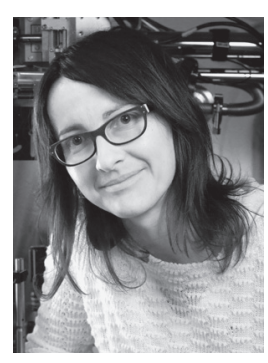

Quantum Nanophotonics

Jelena Vuckovic, Stanford University, USA

Jelena Vuckovic is a Professor of Electrical Engineering and by courtesy of Applied Physics at Stanford University. She received her Ph.D. degree from California Institute of Technology (Caltech) in 2002, and joined Stanford Univ. the same year, first as a postdoctoral scholar and then as a faculty member in 2003. Her research focus is experimental nanophotonics and quantum optics, from fundamental studies of light-matter interaction to applications in quantum technologies, computing, communications, and medicine. Her research has been recognized by numerous awards including the Humboldt Research Award (2010), the Hans Fischer Senior Fellowship (2013), and the Presidential Early Career Award for Scientists and Engineers - PECASE (2007). Vuckovic is a member of the scientific advisory board of the Max Planck Institute for Quantum Optics - MPQ (in Garching, Germany) and of the scientific advisory board of the Ferdinand Braun Institute (in Berlin, Germany).

\section{Plenary Session III \& Awards Ceremony Wednesday, 8 June, 13:00-15:00 \\ Grand Ballroom}

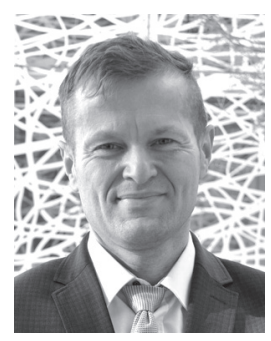

Electrons in Real Time: Tracking and Controlling Motions at the Picometerattosecond Scale

Ferenc Krausz, Max Planck Institute of Quantum Optics; Ludwig-MaximiliansUniversität München, Germany

In 1986, Krausz received a diploma in Electrical Engineering from Budapest University Technology, Hungary and in 1991 received his Ph.D. in Physics from the Vienna University Technology, Austria and was a professor from 1998-2004. In 2003 he became a Director at the Max-Planck-Inst. Quantenoptik, Garching, Germany and later became Professor at LudwigMaximilians-Universität München, Germany. Krausz is currently the Director of the Centre for Advanced Laser Applications, Munich, Germany. He focuses on the research areas of Ultrashort-pulse laser techniques, nonlinear optics, high-field physics, attosecond physics: control and real-time observation of the atomic-scale motion of electrons, light sources and measurement techniques for medical applications. 


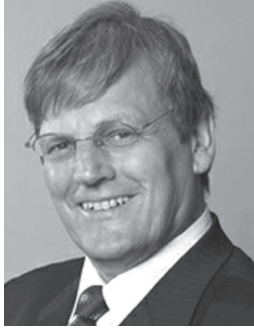

Harvesting Solar Energy with Highest Conversion Efficiencies: Materials Challenges of Record Multijunction Solar Cells

Eicke R. Weber, Fraunhofer Institute for Solar Energy Systems; Albert-Ludwigs University, Germany

Eicke R. Weber is Director of the Fraunhofer Institute for Solar Energy

Systems ISE and Professor for Physics and Engineering at the Albert-Ludwigs-University of Freiburg, Germany. The focus of Weber's research is the analysis of lattice defects in silicon and compound semiconductors, for applications in microelectronics and photovoltaics. Weber obtained his doctorate in Physics from the University of Cologne, Germany in 1976. From 1983-2006 he worked in the Department of Materials Science and Engineering at the University of California Berkeley and since 1991 as Professor of Materials Science. In 1994 he received a US Senior Research Scientist award of the Alexander von Humboldt foundation and served as president of the Alexander von Humboldt Association of America (AvHAA) from 2001-2003. He has received numerous awards including the Award of Merit (Bundesverdienstkreuz am Bande) of the German President, Electronics and Photonics Division Award of the Electrochemical Society ECS, elected Honorary Member of the loffe Physical-Technical Institute of the Russian Academy of Sciences in St. Petersburg, Einstein Award of SolarWorld and received the highly-endowed Zayed Future Energy Prize in Abu Dhabi for his institute, Fraunhofer ISE, from the crown prince of the United Arab Emirates. Since 2010 Prof. Weber has been a member of acatech - the German Academy of Science and Engineering in Berlin. He was elected founding president of the German Energy Storage Association (BVES. Since January 2015 he serves as President of the Association of European Renewable Energy Research Centers EUREC. He is a fellow of the American Physical Society.

\section{IEEE Photonics Society Young Investigator Award}

The IEEE Photonics Society Young Investigator Award honors an individual who has made outstanding technical contribution to photonics (broadly defined) prior to his or her 35th birthday.

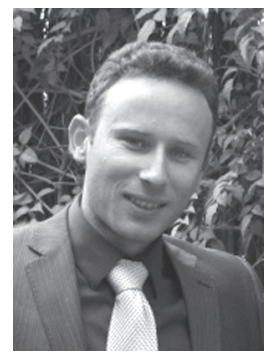

The 2016 award is presented to Igor

Aharonovich from the University of Technology Sydney, Australia for contributions to the development of single photon emitters in wide bandgap materials.

\section{IEEE Photonics Award}

The IEEE Photonics Award, established in 2002, is for outstanding achievements in photonics. The award is sponsored by the IEEE Photonics Society and may be awarded to an individual or a team up to three. The following criteria are considered: outstanding discovery, significant scientific or technological advancement, important invention, impact on the field, and quality of nomination.

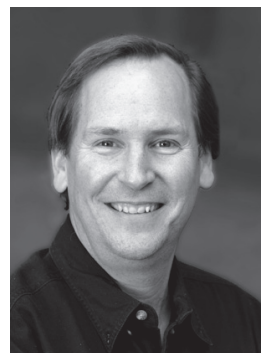

The 2016 recipient is Mark E. Thompson for scientific and technical leadership in the conception, demonstration, and development of phosphorescent materials in organic light-emitting diode (OLED) displays.

\section{OSA Charles Hard Townes Award}

The Optical Society (OSA) established this award in 1980 to honor Charles Hard Townes, whose pioneering contributions to masers and lasers led to the development of the field of quantum electronics. It is given to an individual or a group of individuals for outstanding experimental or theoretical work, discovery or invention in the field of quantum electronics.

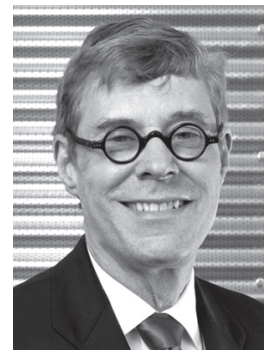

The 2016 recipient is Robert W. Boyd, University of Ottawa, Ottawa, Canada; University of Rochester, USA; and University of Glasgow, UK, for fundamental contributions to the field of nonlinear optics, including the development of methods for controlling the velocity of light, of quantum imaging methods, and of composite nonlinear optical materials.

\section{IEEE Photonics Society 2016 Fellows}

Aaron Hawkins, Brigham Young University, USA

For contributions to optofluidics

William Krupke, Lawrence Livermore National Lab, USA For leadership in laser science and technology

\section{The Optical Society 2016 Fellows}

David Leslie Andrews, University of East Anglia, UK For pioneering research in the formulation of theory based on quantum electrodynamics for the optical interactions of molecules, nonlinear optics and photophysics.

Vanderlei Bagnato, Universidade de São Paulo Instituto de Física de São Carlos, Brazil

For seminal research in light-assisted collisions in laser-cooled atomic gases, including the generation of cold heteronuclear molecules, and for pioneering application of optical therapies to cancer and other diseases in Brazil.

Mikhail A. Belkin, University of Texas at Austin, USA For seminal contributions to photonics technology, spectroscopy and nonlinear optics in the mid-infrared and terahertz spectral range. 
Michael Brodsky, U.S. Army Research Laboratory, USA For significant contributions to the understanding of polarization effects in classical and quantum optical fiber communications.

Stéphane Coen, University of Auckland, New Zealand For in-depth contributions to the understanding of supercontinuum generation in photonic crystal fibers, as well as leading investigations on temporal cavity solitons and identifying their role in microresonator Kerr frequency combs.

Jürgen W. Czarske, Technische Universität Dresden, Germany

For seminal contributions to interferometric and spectroscopic metrology for harsh environments and to adaptive optical systems.

Amir H. Gandjbakhche, National Institutes of Health, USA For leadership and research in areas of non-invasive optical imaging of biological targets, devising quantitative theories, development of methodologies, and designing instrumentation to study biological phenomena.

Ray-Hua Horng, National Chung Hsing University, Taiwan For contributions to green photonics, and in particular for developing high-brightness light-emitting diodes and highefficiency solar cells.

Peter Uhd Jepsen, Danmarks Tekniske Universitet, Denmark For outstanding contributions to applications in terahertz spectroscopy of liquids, molecular crystals and semiconductors.

Sanjay Krishna, University of New Mexico, USA For contributions to the development of infrared detectors and focal-plane arrays.

Ai-Qun Liu, Nanyang Technological University, Singapore For contributions to photonic MEMS/NEMS and optofluidics, and for leadership of optofluidics research and education.

Zhaowei Liu, University of California, San Diego, USA For outstanding contributions to plasmonics and superresolution microscopy.

Yan-qing Lu, Nanjing University, China For significant contributions to microstructured nonlinear optical and electro-optical materials and devices, including crystals and liquid crystals.

John Marsh, University of Glasgow, UK For contributions to quantum and photonic technologies and systems in III-V compound semiconductors.

Takashige Omatsu, Chiba University, Japan

For pioneering contributions in optical vortex lasers and their applications including chiral nanostructure fabrication.

L. Brandon Shaw, U.S. Naval Research Laboratory, USA For contributions to research, development and commercialization of mid-infrared materials, sources and devices.

Günter Steinmeyer, Max Born Institute, Germany For significant contributions to femtosecond pulse measurements and stabilization of the carrier-envelope phase, in particular the feed-forward stabilization method using an acousto-optic frequency shifter.

\section{Incubic/Milton Chang Travel Grant}

The OSA Foundation is pleased to award 10 recipients this year's Incubic/Milton Chang Student Travel Grant, endowed by Milton and Rosalind Chang. The list of recipients can viewed at www.osa.org/foundation.

\section{James P. Gordon Memorial Speakership}

Established in 2014 with the support of the Gordon family, The James P. Gordon Memorial Endowment funds a speakership on Quantum Information and Quantum Optics to a CLEO invited speaker. This speakership pays tribute to Dr. Gordon for his numerous high-impact contributions to quantum electronics and photonics, including the demonstration of the maser.

The recipient receives a $\$ 1,500$ honorarium and their presentation will be recorded and archived in OSA's media library. The contents will serve as an educational resource for the next generation of optics and photonics leaders.

Congratulations to

H. Jeff Kimble, California Institute of Technology, USA.

Kimble will present his talk, Quantum Matter built from Atoms and Photons in Nanoscopic Dielectric Lattices (FM2C.1) at 10:30 on Monday.

\section{Tingye Li Innovation Prize}

The Tingye Li Innovation Prize, established in 2013, honors the global impact Dr. Li made to the field of Optics and Photonics. This prize is presented to a young professional with an accepted paper that has demonstrated innovative and significant ideas and/or contributions to the field of optics. The recipient of this prize receives a $\$ 3,000$ stipend, a special invitation to the Chairs' Reception, and special recognition at the conference.

Congratulations to

Marcus Seidel, Max-Planck-Institute of Quantum Optics, Germany.

Seidel will present his talk, $450 \mathrm{~mW}$ Femtosecond MidIR Source at $\mathbf{8 . 5} \mu \mathrm{m}$ Wavelength (STu3I.6), at 15:00 on Tuesday.

\section{Maiman Student Paper Competition}

The Maiman Student Paper Competition honors American physicist Theodore Maiman for his demonstration of the first working laser and his other outstanding contributions to optics and photonics. It recognizes student innovation and research excellence in the areas of laser technology and electro-optics. The competition results will be announced during the meeting. The award is endowed by a grant from HRL Laboratories LLC, the IEEE Photonics Society and the APS Division of Laser Science and is administered by the OSA Foundation.

Congratulations to our finalists:

Duanni Huang, University of California Santa Barbara, USA

Nils J. Engelsen, Stanford University, USA

Luke Maidment, Heriot Watt University, UK

Deborah Varnell, Princeton University, USA

Mengjie Yu, Cornell University, USA 


\section{Stain-Free Imaging Microscopy for Basic Research and Clinical Applications \\ 6 June, 08:00-12:30}

Salon I \& II, San Jose Marriott

\section{Organizers}

Mikhail Berezin, Washington University in St. Louis, USA

Xingde Li, Johns Hopkins University, USA

For the last century, microscopic analysis of biological samples (i.e. cells and tissues) has been dominated by staining techniques. These traditional labor-intensive and invasive methods rely on treatment of the sample with exogenous contrast agents, such as fluorophores, to improve contrast. Recent advances in optical instrumentation have opened a new era of stain-free techniques that promise to provide strong and quantitative contrast without the use of contrast agents, often obtaining information that cannot be ascertained by conventional methods and enabling label-free optical histology in situ, in vivo and in real time. These include imaging in deep-UV, shortwave, and infrared spectral ranges, as well as photoacoustic, coherent Raman, multiphoton fluorescence, second harmonic time-resolved anisotropy, laserinduced mass-spectrometry, and quantitative phase contrast imaging techniques. The objective of this interdisciplinary symposium is to provide a forum to present and collate significant and exciting studies involving different aspects of and approaches to developing label-free imaging modalities. These approaches span from basic research, such as with live cells, to clinical applications, including tissue histology and in vivo pathology.

\section{Invited Speakers}

Rohit Bhartia, Jet Propulsion Laboratory, California Institute of Tech., USA, Smoke, Mirrors, and Black Boxes: Imaging the Invisible World

Viktor Gruev, Washington Univ. in St. Louis, USA, Bio-inspired Polarization Imaging Sensor for Label-Free Applications

Myung Kim, University of South Florida, USA, Holographic Techniques for Biomedical Microscopy

Richard Levenson, Univ. of California Davis Medical Center, USA, Slide-free (but not necessarily stain-free) Microscopy via UV Excitation

Gabriel Popescu, Univ. of Illinois at Urbana-Champaign, USA, Gradient Light Interference Microscopy (glim) of Optically Thick Specimens

\section{Ultrafast Dynamics in Solids}

6 June, 16:00-18:00; 7 June, 08:00-10:00

$210 D / 212 D$

\section{Organizers}

Hanieh Fattahi, Max-Plank-Institut für Quantenoptik, Germany Nick Karpowicz, Max-Plank-Institut für Quantenoptik,

Germany

François Légaré, INRS-ÉMT, Canada

Olga Smirnova, Max-Born-Institut Berlin, Germany

Over the recent years, ultrafast laser technologies have enabled the generation of ultrashort light pulses up to the hard X-ray spectral range. Together with large scale X-ray Free Electron Laser facilities, researchers have now access to a variety of tools to probe ultrafast processes in matter, with atomic scale and femtosecond to attosecond temporal resolution. Furthermore, recent studies have demonstrated that high harmonic generation, the key process to generate isolated attosecond pulses, can be extended to the solid state paving the way to probe ultrafast electronic processes in condensed matter. This symposium will address the most recent advances in the field of ultrafast dynamics in solids, including, but not restricted to, the topics of attosecond dynamics in condensed matter, attosecond surface science, ultrafast phase transitions in correlated materials, ultrafast magnetization dynamics, high harmonic generation from solids and future applications.

\section{Invited Speakers}

Joachim Burgdörfer, Technische Universität Wien, Austria, Ab-initio Simulations of Strong-field Processes in Widebandgap Insulators

Alexander X. Gray, Temple University, USA, Dynamic Control of Fundamental Physical Interactions in Strongly-Correlated Oxides

Fabian Langer, Universität Regensburg, Germany, Terahertzdriven High Harmonic Generation in Bulk Crystals

Mark Stockman, Georgia State University, USA, Adiabatic Control of Solids by Strong Ultrafast Optical Fields

Giulio Vampa, University of Ottawa, Canada, Linking High Harmonics from Gases and Bulk Solids

Clemens von Korff Schmising, Technische Universität Berlin, Germany, Probing Ultrafast Magnetization Dynamics with High-Harmonic Magnetic Circular Dichroism

\section{Nanophotonics with 2D Materials}

7 June, 13:00-18:00

$210 \mathrm{~F}$

\section{Organizers}

Nick Vamivakas, University of Rochester, USA

Xiadong Xu, University of Washington, USA

New ideas continuously emerge at the intersection of materials science, nano-optics and condensed matter physics to address fundamental and technological challenges in nanophotonics. Particularly important is the ability to engineer nanophotonic devices that support confined excitonic and optical modes that result in enhanced light-matter interaction. A current, novel and attractive approach to realize confined electronic resonances are atomically thin materials - both semiconductors and semi-metals. And, although graphene has been extensively studied in the last decade, only recently has the focus turned to atomically thin semiconductors. The ability to seamlessly integrate atomically thin materials, and their van der Waal's heterostructures, with optical cavities and waveguides is providing a novel pathway to expand the performance and functionality of nanophotonic devices. Equally exciting is the possibility for devices that leverage the properties of the two-dimensional material's electronic structure such as the spin or valley degree-of-freedom. Nano-

All conference locations are in the San Jose Convention Center unless otherwise noted. 
photonic devices based on these degrees of freedom would find application in both spintronics and the emerging area of valleytronics. Finally, just recently it was realized that atomically thin semiconductors also host quantum dot like defects. This observation will open a pathway toward leverage 2D materials for integrated quantum photonics applications. This symposium will explore emerging areas of nanophotonics that leverage the unique properties of 2D materials. Focus areas will span novel classical and quantum nanophotonic devices based on these materials.

\section{Invited Speakers}

Palash Bharadwaj, Rice University, USA, Antenna-coupled Optoelectronics with Two-dimensional Materials

Chitraleema Chakraborty, University of Rochester, USA, Quantum Nanophotonics with Localized Excitons in Atomically Thin Semiconductors

Xiangfeng Duan, California NanoSystems Institute, USA, Vertically Stacked Heterostructures for Tunable Photonic Devices - from 2D Materials to Hybrid Perovskites

John Schaibley, University of Washington, USA, Nonlinear Spectroscopy of Valley Excitons in 2D Semiconductors and Heterostructures

Jie Shan, Pennsylvania State University, USA, Optical Imaging of the Valley Hall Effect In Two-Dimensional Semiconductors

\section{Advances in Single-Photon Detector Technologies}

8 June, 08:00-10:30; 15:30-17:30

$210 \mathrm{C}$

\section{Organizers}

Joshua C. Bienfang, NIST, USA

Francesco Marsili, Jet Propulsion Laboratory, USA

Alan Migdall, Joint Quantum Institute at University of Maryland, USA; NIST, USA

Matthew Shaw, Jet Propulsion Laboratory, USA

Single-photon detectors (SPDs) operate at the fundamental limit of electromagnetic signal strength and represent an enabling technology for a wide range of applications spanning both the classical and quantum regimes, including quantum information science, quantum and classical optical communications, advanced metrology, quantum optics, molecular biology, LIDAR, and environmental sensing. In all of these applications, the performance of the detectors play a fundamental role in the performance of the overall system, and as the state of the art in these fields has advanced, so have the performance requirements of the constituent detectors. This has led to a concerted effort to advance singlephoton detection technologies to achieve higher efficiency, lower noise, higher speed and timing resolution, as well as to improve other properties, such as photon number resolution, imaging, and sensitivity to lower energy photons. This symposium focuses on recent advances in single-photon-detection technologies as motivated by the demands of the state of the art in classical sensing and quantum-based technologies.

\section{Invited Speakers}

Karl Berggren, MIT, USA, Superconducting Nanowire SinglePhoton Detectors and Nanowire-Based Superconducting On-Chip Electronics

Marissa Giustina, 10OQI - Austrian Academy of Sciences, Austria, A Significant-loophole-free Test of Local Realism

Ronald Hanson, QuTech - Delft University of Technology, Netherlands, From the first loophole-free Bell test to a Quantum Internet

Ben Mazin, University of California Santa Barbara, USA, Microwave Kinetic Inductance Detectors for Single Photon Counting with Energy Resolution

Krister Shalm, NIST, USA, A Strong Loophole-free Test of Local Realism

Alberto Tosi, Politecnico di Milano, Italy, Short-gate techniques for high-speed photon counting with InGaAs/InP SPADs

\section{Symposium Celebrating 20 Years of Photonic Crystal Fiber}

8 June, 08:00-10:00; 15:30-17:30

211B

\section{Organizers}

Amir Abdolvand, Max Planck Institute for the Science of Light, Germany

David Novoa, Max Planck Institute for the Science of Light, Germany

John Travers, Max Planck Institute for the Science of Light, Germany

The control of diffraction through tailoring the transverse microstructure of photonic crystal fibers (PCF), first demonstrated 20 years ago, enables the confinement of light in a far more versatile way than possible with conventional optical fibers. It is possible to both significantly enhance or reduce the nonlinear interaction of the guided field with the PCF, and also widely customize the waveguide dispersion. Hollowcore PCFs extend these benefits to fibers filled with liquids, atomic-vapors, gases or even plasma. The use of different glass compositions to fabricate PCFs offers an extra degree of freedom, especially for expansion into the mid-infrared and deep ultraviolet regimes. These features have been used in fundamental research and for a large variety of applications. Examples include: supercontinuum generation, high-capacity data transmission, quantum-optics, vacuum ultraviolet generation, high-power fiber laser amplifiers, high-power beam delivery, and intense pulse compression. In this symposium we intend to combine reviews of previous work on PCF and its applications, with an optimistic outlook to what lies ahead.

\section{Invited Speakers}

Jens Limpert, Friedrich Schiller University Jena, Germany, High Power Pulse Generation and Compression with Photonic Crystal Fibers

Arnaud Mussot, Université Lille 1, France, Title to be Determined

Jeff Nicholson, OFS Optics, USA, Advances in PolarizationMaintaining, Single-Mode, Hollow-Core Fibers 
David Richardson, Optoelectronics Research Centre University of Southampton, UK, Optical Communications using Microstructured Optical Fibers

Philip Russell, Max-Planck-Institute for the Science of Light, Germany, A Quarter Century of Photonic Crystal Fibre

William Wadsworth, University of Bath, UK, Useful Light from Photonics Crystal Fibres

Panel - PCF in Industry

Fetah Benabid, Xlim Research Insititute, France

Anatoly Grudinin, Fianium, UK

Alkeskjold Thomas, NKT Photonics Inc, Denmark

\section{Optical Signal Processing with Phase- \\ Sensitive Amplifiers}

9 June, 14:00-18:30

$210 F$

\section{Organizers}

Michael Galili, Danmarks Tekniske Universitet, Denmark Leif Johansson, Freedom Photonics, USA

Michael Vasilyev, University of Texas at Arlington, USA

All-optical processing of high-speed signals carrying information encoded in both amplitude and phase is of paramount importance for communications, computing, imaging, and other applications. Access to and manipulation of the optical phase information requires coherent signal processing capabilities. Recently, such capabilities have been realized by nonlinear-optical means - namely, by using phase-sensitive parametric amplifiers (PSAs). Only a decade ago, the PSAs were a domain of fundamental quantum optics research. Since then, extensive work has been done on bridging the quantum-classical divide and bringing the unique capabilities of the PSA technology to the forefront of today's high-speed signal processing. Rapidly advancing, this technology has been extended to very sophisticated multilevel modulation formats, generation of high-quality optical frequency combs, new promising material platforms for on-chip implementations, etc. This symposium will provide a forum for discussing the recent advances in phase-sensitive amplification technology and the new horizons in coherent signal processing opened by it.

\section{Invited Speakers}

Peter Andrekson, Chalmers University, Sweden, Optical Fiber-based Phase Sensitive Amplifiers and their Application in Optical Communication Systems

Ping Piu (Bill) Kuo, University of California at San Diego, USA, Frequency-domain Signal Processing based on Comb-generating Parametric Mixers

Prem Kumar, Northwestern University, USA, Spatially-multimode phase-sensitive parametric amplification: investigation of eigenmodes with Gaussian-beam Pumping

Wangzhe Li, University of California at Santa Barbara, USA, Investigation of an Integrated Photonic Dual-Pumped Phase-Sensitive Amplifier based on a Highly Saturated Semiconductor Optical Amplifier

Colin McKinstrie, Applied Communication Sciences, USA, Principles and Applications of Phase-sensitive Amplifiers
Periklis Petropoulos, University of Southhampton, UK, Applications of Nonlinear Parametric Effects for Advanced Processing of Optical Signals

Young Zhang, CUDOS-University of Sydney, Australia, Phase-sensitive Amplification in Silicon and Chalcogenide Waveguides

\section{Advances and Opportunities in Astrophotonics \\ 10 June, 08:00-12:00 \\ Salon I \& II, San Jose Marriott}

\section{Organizers}

Lucas Labadie, Physics Institute, University of Cologne, Germany

Robert Thomson, Heriot-Watt University, UK

Michael Withford, Macquarie University, Australia

The field of astrophotonics seeks to apply photonic concepts to astronomical instrumentation, with the overall aim of enabling improved astronomical instruments with unprecedented precision, flexibility, multiplex gain. But although astronomy can leverage the massive investment that has been made in photonic technologies over the last half a century, the field of astrophotonics is also driving the development of entirely new photonic technologies. A good example of this is the "photonic lantern" which enables the efficient coupling of incoherent multimode light to single mode photonic devices, and the development of ultra-broadband fibre Bragg-gratings of unprecedented complexity, in order to block undesirable atmospheric spectral lines from entering a spectrograph. These technologies are now finding spin-off applications in areas such as telecommunications and remote sensing, and it is clear that astrophotonics is both an utiliser and driver of advanced photonic technologies. Other important examples are found with the use of high gain fiber scramblers for high-resolution spectrographs, frequency combs for unprecedented spectral calibration, or integrated active/passive photonics functions for stellar Interferometry.

\section{Invited Speakers}

Thierry Fusco, Onera Paris, France, Adaptive Optics in the Extremely Large Telescope Area: New Requirements, New Concepts and New Challenges

Olivier Guyon, Subaru Telescope - National Astronomical Observatory of Japan, Japan, Where are our Closest Neighbours? Looking for Life on Nearby Exoplanets

Timothy Morris, University of Durham, UK, Astrophotonics and Adaptive Optics - A Match Made in the Stars?

Mike Shao, Jet Propulsion Labs, USA, Big Science in Small Packages: a Constellation of Cubesats to Search for Near Earth Asteroids

Peter Tuthill, University of Sydney, Australia, Photonic Stellar Interferometry: to the Diffraction Limit and Beyond 


\section{Mid-IR Lasers: Advancing from Research Topic to Application}

10 June, 08:00-12:30

212B

\section{Organizers}

Kenneth L Schepler, Univ. of Central Florida, USA Irina Sorokina, Norwegian Univ. of Science and Technology, Norway

Mid-IR $(2-5 \mu \mathrm{m})$ laser classes are just now beginning to experience emerging maturity and to transition to commercial availability. These laser classes include quantum cascade lasers, interband cascade lasers, transition-metal lasers and rareearth lasers. A variety of forms such as fibers, waveguides, stripes, quantum dots and traditional bulk crystals have been demonstrated. Continuous wave, Q-switched, modelocked operation and broadband tunability have also been demonstrated. However, the laser of choice for specific applications is dictated by which forms and operating characteristics are relevant. No particular laser class is best for all applications. This symposium will review the state of the art in mid-IR laser development, present latest laser developments, present demonstrations of new applications of infrared sources, and discuss the niches where particular kinds of mid-IR lasers are best.
Invited Speakers

Timothy Day, Daylight Solutions, Inc, USA, Quantum Cascade Lasers: A Platform Technology for Today's Marketplace

Jihong Geng, AdValue Photonics, USA, Application of Midinfrared Fiber Laser Sources

Sergey Mirov, University of Alabama at Birmingham, USA, Transitioning $\mathrm{Cr}^{2+}$ and $\mathrm{Fe}^{2+}$ doped $\mathrm{Zn}$ Chalcogenide Laser Technology to Commercial Products - Lessons Learned

C. Kumar Patel, Pranalytica Inc., USA, Sub Microsecond Tuning and Rapid Transmission and Standoff Detection Using Quantum Cascade Lasers

Frank Tittel, Rice University, USA, Recent Advances of Midinfrared Compact, Field Deployable Sensors and their Real World Applications in the Petrochemical Industry, Atmospheric Chemistry and Security 


\section{Applications \& Technology Topical Reviews}

\author{
Extreme Ultraviolet and Soft X-Ray Sources \\ and Applications \\ 6 June, 08:00-12:30 \\ $212 \mathrm{~A}$ \\ Organizers \\ Carmen S. Menoni, Colorado State University, USA \\ Alexander Ershov, Cymer, USA
}

The Topical Review on Ultraviolet/Extreme ultraviolet lasers and applications will highlight advances in the demonstration of compact EUV sources, including plasma based lasers and higher harmonics, the characterization of these sources and their applications. The session will also emphasize advances in sources for extreme ultraviolet lithography and metrology which provide the foundations for the printing of next generation semiconductor chips.

\section{Tutorial Speaker}

Jorge Rocca, Colorado State University, USA, Table-top Soft X-Ray Lasers

Invited Speakers

Annie Klisnick, CNRS, Université Paris-Saclay, France, Characterization of Spectral and Temporal Properties of Plasmabased XUV Laser Pulses

Margaret Murnane, University of Colorado, USA, New Developments in High Harmonic Generation and Applications in Imaging and Materials Science

Patrick Naulleau, Lawrence Berkeley Lab, USA, Metrologies

supporting EUV Lithography

Eléonore Roussel, ELETTRA Sincrotrone Trieste, Italy, The FERMI Free-Electron Laser

Regina Soufli, Lawrence Livermore National Lab, USA, EUV/X-ray Multilayer Optics: Meeting the Challenges of Next-Generation Applications

\section{Advances in Laser-based Remote Sensing}

6 June, 13:30-18:00

$212 \mathrm{~A}$

\section{Organizer}

Fabio Di Teodoro, Space and Airborne Systems - Raytheon Company, USA

Active remote sensing generally refers to the integration of a laser source; optical beam formatting, direction, and collection apparatuses; photodetectors; and data processing infrastructures to perform a broad spectrum of reconnaissance, imaging, and standoff analysis tasks including range finding of still or moving targets, terrain topography, detection of airborne biological/chemical species, wind speed measurements, etc.

This topical review addresses cutting-edge research and development of active remote sensors from a system standpoint to include new laser transmitters, detectors, and advanced sensing concepts. Particular emphasis will be placed on long-range sensors intended for deployment in airborne and space platforms. Such systems must address many technological challenges devoted to the maximization of the laser transmitter pulse energy/power; minimization of size, weight, and power consumption (SWaP); increase in rugged-

ness and support for operation in thermo-mechanical harsh environments; and improvement of long-term reliability.

Invited Speakers

Brian Aull, MIT Lincoln Lab, USA, Single-Photon-Sensitive

Solid-State Image Sensors for Flash Lidar

Guillaume Canat, ONERA, The French Aerospace Lab, France, High Peak Power Single-Frequency MOPFA for Lidar Applications

Maurice Halmos, Raytheon, USA, Coherent Detection with Photon Counting GMAPD Receiver

Gary Kamerman, Fastmetrix, USA, Title to be Determined Anthony Yu, NASA Goddard Space Flight Center, USA, NonTopographic Space-Based Laser Remote Sensing

\section{Supercontinuum Laser Technology and its Applications}

6 June, 13:30-18:00

211D

Organizer

John Clowes, Fianium Limited, UK

Supercontinuum (White Light) Lasers have recently emerged from the laboratory into commercial light sources used in applications from scientific research to manufacturing, defence and security. Decades of R\&D in materials science, photonics components and laser physics have led us to this stage, yet the performance limits of this unique laser technology continue to be pushed by the scientific community and laser manufacturers.

\section{Invited Speakers}

Mohammed Islam, University of Michigan, USA, Swir SuperContinuum Laser for Active Illumination in Hyper-Spectral Imaging

James Stone, University of Bath, UK, The Rising of the Supercontinuum

\section{Neurophotonics}

7 June, 13:30-18:00

Salon I \& II, San Jose Marriott

\section{Organizer}

Kishan Dholakia, Univ. of St. Andrews, UK

Neurophotonics has emerged as very exciting contemporary challenge that aims to merge interface cutting edge photonics technology and neuroscience. Neurophotonics embraces both invasive and non-invasive methods that can be applied right from single cells to whole animal studies. The approaches promise a paradiogm shift in the way we may image, probe and interrogate functional activity in the brain. Grand challenges in this field include recording and manipulating the activity of multiple cells in the brains of live animals using light. Major opportunites exist for using innovations in photonics to address these questions that ultimately may impact our view of the functioning of the brain and the emergence of diseases such as dementia.

This topical review will cover a whole host of methods in this expanding area that may include advanced forms of single 
photon and multiphoton imaging including light sheet imaging, diffuse correlation spectroscopy, near infrared spectroscopy, novel probes and light sources.

\section{Invited Speakers}

Meng Cui, Purdue University, USA, Wavefront Control for Deep Tissue Fluorescence Microscopy

Chris Xu, Cornell University, USA, Optical Technology Development for Mouse Brain Imaging

\section{Lasers in Materials Processing}

7 June, 13:30-17:45

$212 \mathrm{~A}$

\section{Organizer}

Arnold Gillner, Fraunhofer Institute for Laser Technology Aachen, Germany

The Topical Review session on Lasers in Material Processing will highlight recent laser materials processing applications from automotive, aerospace, electronics, medical and other application fields. With an emphasis on industrial laser processing technologies, like welding, cutting, drilling, surface treatment, materials modifications and ablation, the conference will give a broad overview on the possibilities of high accuracy and high speed laser processing. The session will include laser applications with cw-Lasers, like fiber and disc laser with new properties, lasers with adapted wavelengths as well as short and ultra short pulsed lasers in the ps- and $\mathrm{fs}$-regime. Actual industrial proven processes will be also presented as upcoming applications where new lasers and beam guiding systems will generate well desired functionalities and materials properties. Contributions from interaction phenomena as well as particular industrial installations will show, how fundamental know how research is necessary for a successful laser integration and materials processing.

\section{Invited Speakers}

Klaus Bergner, University of Jena, Germany, Time-resolved Microscopy for Optimizing In-volume Glass Processing using Ultra Short Laser Pulses

Dirk Petring, Fraunhofer ILT, Germany, Laser Beam Cutting A Mature and Still Advancing Laser Application Becomes Calculable

\section{Photoacoustic Imaging: From Promises to Maturation}

9 June, 14:00-18:00

Salon I \& II, San Jose Marriott

\section{Organizer}

Wiendelt Steenbergen, Universiteit Twente, Netherlands

Photoacoustic/optoacoustic imaging is a long-time promising technology for biomedical imaging. However, despite almost 2 decades of research, photoacoustics is still not a clinically accepted imaging modality for any application. It is difficult to beat the established imaging modalities, and to convince medical users and decision makers to use the technology. What is needed to enter the clinic are both a technological maturation and evidence of the medical added value for certain 'killer applications'.

This topical review session will focus on the technological aspects of casting photoacoustic imaging into implementations that are robust and affordable. We will particularly focus on the aspects of multimodality, system integration, light source development and quantitative imaging. The session will provide a mixture of invited and contributed talks. Contributed papers are invited on topics that fit in one of the above aspects, such as: novel light sources, increase of penetration depth, reduction of imaging artefacts, quantification, integration with existing modalities, and novel systems for use on humans.

\section{Invited Speakers}

Emad M. Boctor, Johns Hopkins University, USA, Title to be Determined

Martin Frenz, University of Berne, Switzerland, Multimodal Biomedical Optoacoustic Imaging

Andreas Kohl, Quantel Laser, France, A Diode Laser Sources for Pa Imaging: An Enabling Technology for Portable Devices

Tsuyoshi Shiina, University of Kyoto, Japan, Innovative Photoacoustic Imaging Technology towards the Creation of Vascular Health Science

\section{Lasers in Additive Manufacturing - Fundamentals and Applications \\ 10 June, 08:00-11:45 \\ $212 \mathrm{~A}$}

\section{Organizers}

Ruth Houbertz, Multiphoton Optics GmbH, Germany Mangirdas Malinauskas, Laser Research Center of Vilnius University, Lithuania

The Topical Review on Lasers in Additive Manufacturing Fundamentals and Applications will bring together representatives from industries and academia working with this futureoriented enabling technology. Many efforts have been made to develop light sources in order to continuously increase their application potential. The availability of extremely stable lasers provides an enormous flexibility in additive processing. Particularly ultrashort pulse lasers have enabled highprecision 3D optical printing with a precise control of spatial features in metals, glasses, ceramics, and polymers. Recent advances proves the possibility to tune the modification type and produce multimaterial monolithic structures paving the way from $3 D$ to $4 D$ printing where the properties of a material can be tuned by the exposure conditions.

Presentations will shed light on many different aspects of materials' additive processing, ranging from nano- via micro- to macro-processing with giving a special emphasis on nano- and micro-fabrication. Fundamentals of light-matter interaction and basic modification mechanisms together with fabrication throughput and repeatability issues in freeform 3D structure formation will be addressed. A variety of exciting applications from different application fields such as microoptics, optical communications, metamaterials, plasmonics, biomedical and microfluidic devices, and implants, will be presented.

\section{Invited Speakers}

Saulius Juodkazis, Swinburne University of Technology, Australia, Writing of Bio-Compatible Silk Patterns: 3D Laser Nano-Printing

Satoshi Kawata, Osaka University, Japan, 3D Nano-fabrication of Metallic Nano-structures

Markus Riester, Maristechcon, Germany, Additive Manufacturing for Optical Network Components

All conference locations are in the San Jose Convention Center unless otherwise noted. 


\section{Short Courses}

\section{Short Course Chairs}

Sasan Fathpour, CREOL, Univ. of Central Florida, USA

Ben Eggleton, Univ. of Sydney, Australia

The CLEO: 2016 Short Course Program includes a range of topics at a variety of educational levels. Widely recognized experts in industry and academia lead attendees in building skills and/or achieving new insight, and the small-classroom setting provides a tremendous, interactive learning opportunity. Short Courses are an excellent opportunity to learn about new products, cutting edge technology and vital information at the forefront of the laser science and electro-optics fields.

Certificates of Attendance are available for those who register and attend a course. You may request a certificate upon completion of the online course evaluation. If you have any questions about receiving a Certificate of Attendance or completing the course evaluation, please email shortcourses@cleoconference.org with your name and course name(s).

\section{Sunday, 5 June 2016}

\section{8:30-12:30}

SC149: Foundations of Nonlinear Optics Instructor: Robert Fisher, R. A. Fisher Associates, USA

SC221: Nano Photonics: Physics and Techniques Instructor: Axel Scherer, Caltech, USA

SC301: Quantum Cascade Lasers: Science, Technology, Applications and Markets

Instructor: Federico Capasso, Harvard Univ., USA

SC361: Coherent Midlnfrared Sources and Applications Instructor: Konstantin Vodopyonov; CREOL, Univ. Central Florida, USA

\section{3:30-16:30}

SC403: NanoCavity Quantum Electrodynamics and Applications Instructor: Jelena Vuckovic, Stanford Univ., USA

\section{3:30-17:30}

SC157: Laser Beam Analysis, Propagation, and Shaping Techniques

Instructor: James Leger; Univ. of Minnesota, USA

SC378: Introduction to Ultrafast Optics

Instructor: Rick Trebino, Georgia Institute of Technology, USA

SC396: Frontiers of Guided Wave Nonlinear Optics

Instructor: Ben Eggleton, Univ. of Sydney, Australia
Monday, 6 June 2016

09:00-12:00

SC362: Cavity Optomechanics: Fundamentals and Applications of Controlling and Measuring Nano- and Micro-mechanical Oscillators with Laser Light Instructor: Tobias Kippenberg, Ecole Polytechnique Federale de Lausanne, Switzerland

NEW SC376: Plasmonics Instructor: Mark Brongersma, Stanford Univ., USA

SC440: How to Communicate High-Tech to the Market - A Marketing and PR Primer NEW

Instructor: Andreas Thoss, THOSS Media GmbH, Germany

$12: 30-15: 30$

SC424: Optical Terahertz Science and Technology Instructor: David G. Cooke; Dept. of Physics, McGill University, Canada

NEW SC439: Attosecond Optics Instructor: Zenghu Chang, CREOL, Univ. of Central Florida, USA

Tuesday, 7 June 2016

\section{2:00-15:00}

SC352: Introduction to Ultrafast Pulse Shaping-Principles and Applications

Instructor: Marcos Dantus, Michigan State Univ., USA

SC410: Finite Element Modeling Methods for Photonics and Optics

Instructor: Arti Agrawal, City Univ., UK

\section{2:00-16:00}

SC270: High Power Fiber Lasers and Amplifiers Instructor: W. Andrew Clarkson, Optoelectronics Res. Ctr., Univ. of Southampton, UK

\section{NEW SC438: Photonic Metamaterials} Instructor: Nader Engheta, University of Pennsylvania, USA

All conference locations are in the San Jose Convention Center unless otherwise noted. 


\section{Short Course Descriptions}

Courses are listed by date and time. Complete course descriptions are available at www.cleoconference.org/ shortcourses.

Instructors have designated a course level to help you select a course that best fits your educational background and goals.

Beginner: No background or minimal training is necessary to understand course material.

Advanced Beginner: Basic understanding of topic is necessary to appreciate course material.

Intermediate: Prior knowledge of topic is necessary to follow course material.

\section{SC149 - Foundations of Nonlinear Optics}

Sunday, 05 June, 08:30-12:30

Robert Fisher, R. A. Fisher Associates, USA

Short Course Level: Beginner

Short Course Description: This introductory and intermediate level course provides the basic concepts of nonlinear optics. Although some mathematical formulas are provided, the emphasis is on simple explanations. It is recognized that the beginning practitioner in nonlinear optics is overwhelmed by a constellation of complicated nonlinear optical effects, including second-harmonic generation, optical Kerr effect, self-focusing, self-phase modulation, self-steepening, fiberoptic solitons, chirping, stimulated Raman and Brillouin scattering, and photorefractive phenomena. It is our job in this course to demystify this daunting collection of seemingly unrelated effects by developing simple and clear explanations for how each works, and learning how each effect can be used for the modification, manipulation or conversion of light pulses. Examples will address the nonlinear optical effects that occur inside optical fibers and those that occur in liquids, bulk solids, and gases.

\section{Short Course Benefits:}

This course should enable you to:

- Explain and manipulate the Slowly-Varying Envelope Approximation (SVEA)

- Recognize what nonlinear events come into play in different effects

- Appreciate the intimate relationship between nonlinear events which at first appear quite different

- Discuss how a variety of different nonlinear events arise, and how they affect the propagation of light

- Describe how wavematching, phase-matching, and index matching are related

- Summarize how self-phase modulation impresses "chirping" on pulses

- Explain basic two-beam interactions in photorefractive materials

- Develop an appreciation for the extremely broad variety of ways in which materials exhibit nonlinear behavior"
Short Course Audience: Although we start at the very beginning of each topic, we move quite rapidly in order to grasp a deep understanding of each topic. Therefore, both beginners and intermediates will benefit greatly from this course. The material will be of interest to graduate students, to researchers, to members of the legal profession, to experts who are just transferring to this field, to managers, and to anyone else who just wants to learn how nonlinear optics works. This course will give an excellent nonlinear optics foundation for those feeling the need so they can also take any of the following more specialized nonlinear optics courses at this CLEO conference such as SC396; SC378, SC270, SC410, and SC352.

\section{SC221 - Nano Photonics: Physics and Techniques} Sunday, 05 June, 08:30-12:30

Axel Scherer, Caltech, USA

Short Course Level: Intermediate

Short Course Description: Students will learn about the applications of printed and integrated optical devices. In particular, optical microcavities and vertical cavity lasers, silicon photonics and plasmonic systems will be introduced and compared. Integrated opto-electronic and opto-fluidic systems for communications and biomedical sensing will be compared.

\section{Short Course Benefits:}

This course should enable you to:

- Compare dielectric (total internal reflection and Bragg reflectors) with metallic (surface plasmon) geometries for confining and guiding light

- Identify opportunities for using printed optical systems in silicon (silicon photonics)

- Describe methods for creating quantum-mechanical systems from optical nanostructures

- Design lithographically defined micro- and nanocavities for resonators and lasers

- Define applications of printed optics in biochemical sensing

- Summarize the evolution of printed optical integrated circuits and devices, such as modulators and switches

- Determine the applications of interdisciplinary integration of optics with electronics and fluidics

- Describe optical performance of semiconductor structures when these are made with nanoscale dimensions

Short Course Audience: This course is designed for participants with interest in miniaturizing optical devices. Methods of microfabricating dielectric and plasmonic devices will be described, along with examples of their applications and description of future opportunities. 
SC361 - Coherent Midlnfrared Sources and

\section{Applications}

Sunday, 05 June, 08:30-12:30

Konstantin Vodopyonov; CREOL, The College of Optics \&

Photonics, Univ. Central Florida

\section{Short Course Level: Intermediate}

Short Course Description: The course covers fundamental principles of mid-IR generation and reviews different approaches for producing coherent light in this important yet challenging spectral region. It examines different state-of-theart techniques from diverse areas of photonics that include solid-state lasers (based on rare earth and transition metals), fiber lasers, semiconductor lasers (including intra- and intersubband cascade lasers), laser sources based on nonlinear optical frequency downconversion, Raman sources and others. The course reviews several emerging technologies such as supercontinuum generation in highly nonlinear fibers and waveguides, as well as frequency combs. Several important mid-IR applications will be reviewed including trace molecular detection, coherent spectroscopy using frequency combs, and some medical applications.

\section{Short Course Benefits:}

This course should enable you to:

- Get a clear idea of existing laser sources in the midIR spectral region $(2-20 \mu \mathrm{m})$ and understand their operational principles, as well as advantages and disadvantages.

- Distinguish between different operational regimes, from continuous-wave to few optical cycle regime.

- Distinguish between broadband and narrow-band sources, as well as between a supercontinuum and a frequency comb sources.

- Learn about new applications of mid-IR coherent sources, from trace molecular detection and remote sensing to ultrafast spectroscopy and attosecond physics.

- Identify what kind of laser source you need for your particular application.

Short Course Audience: Students, academics, researchers and engineers in various disciplines who require a broad introduction to the subject and would like to learn more about the state-of-the-art and upcoming trends in mid-infrared coherent source development and applications. Undergraduate training in either engineering or science is assumed.

\section{SC403 - NanoCavity Quantum Electrodynamics and Applications}

Sunday, 05 June, 13:30-16:30

Jelena Vuckovic, Stanford Univ., USA

\section{Short Course Level: Beginner}

Short Course Description: Strong localization of light in nanophotonic structures leads to enhanced light-matter interaction, which can be employed in a variety of applications, ranging from improved (higher speed, lower threshold) optoelectronic devices, to biophotonics, quantum information and low threshold nonlinear optics.
In particular, quantum dots in optical nanocavities are interesting as a test-bed for fundamental studies of such lightmatter interaction (cavity quantum electrodynamics - QED), as well as an integrated platform for information processing. As a result of the strong field localization inside of sub-cubic wavelength volumes, they enable very large emitter-field interaction strengths (vacuum Rabi frequencies in the range of 10 's of GHz - a few orders of magnitude larger than in atomic cavity QED). In addition to the study of new regimes of cavity QED, this can also be employed to build devices for quantum information processing, such as ultrafast quantum gates, nonclassical light sources, and spin-photon interfaces. Beside quantum information systems, many classical information processing devices greatly benefit from the enhanced light matter interaction in such structures; examples include alloptical switches operating at the single photon level, electrooptic modulators controlled with sub-fJ energy and operating at $\mathrm{GHz}$ speed, and lasers with threshold currents of 100nA.

This course will introduce cavity QED (e.g., strong and weak coupling regimes, Purcell effect, etc.), with particular emphasis on semiconductor nanocavities. We will also describe state of the art in solid state cavity QED experiments and applications.

\section{Short Course Benefits:}

This course should enable you to:

- Explain light matter interaction in optical nanostructures

- Discuss state of the art in solid state cavity QED

- Identify benefits of employing nano-cavity QED for certain applications

Short Course Audience: Scientists and engineers interested in cavity QED and nanophotonic devices in general. Some background in electromagnetics, quantum mechanics, and optoelectronics is helpful, but not required.

\section{SC157 - Laser Beam Analysis, Propagation, and Shaping Techniques}

Sunday, 05 June, 13:30-17:30

James Leger; Univ. of Minnesota, USA

\section{Short Course Level: Beginner}

Short Course Description: The performance of conventional high power lasers is often compromised by one or more physical effects, limiting the maximum power that can be obtained from a single lasing element. To increase the power from these individual elements, laser beam combining can be employed to convert the outputs from several lower-power modules into a single, high-power beam. This short course establishes general beam combining principles relevant to all laser systems, and emphasizes the limits that are achievable with differ approaches. The practicing engineer and technical manager will be introduced to a wide variety of beam combining methods. Incoherent beam combining attempts to maximize the radiance of an array of incoherent sources. The theoretical limits of this approach will be derived, and a design methodology developed to achieve maximum radiance. Spectral and polarization beam combining techniques employ wavelength and polarization sensitive elements to sum laser power. Several practical issues of this technique will be discussed, and specific systems described. Coherent beam combining is introduced by exploring methods 
of establishing mutual coherence across laser arrays. The properties and characteristics of these coherent techniques are quantitatively analyzed using simple modal theories. Methods of converting arrays of coherent beams into a single beam are explored, and the sensitivity of these approaches to path length errors investigated. Real-world examples will be used as case studies to illustrate design principles. This offering of the course will make use of recently developed material on coherent beam combining architectures.

\section{Short Course Benefits:}

This course should enable you to:

- Describe the requirements for laser beam combining of all types.

- Estimate the optimum brightness enhancement achievable from incoherent combining.

- Design an ideal incoherent beam combiner.

- Design spectral beam combiners and estimate performance limitations.

- Compare different architectures for establishing mutual coherence across laser arrays.

- Determine the effects of path length errors on beam combining performance.

- Design optical systems to convert coherent arrays of laser beams into a single beam.

- Describe the performance characteristics of several laser systems that utilize beam combining.

Short Course Audience: The course is designed for students, engineers, scientists and technical managers who are interested in understanding the basics of laser beam combining. No advanced knowledge of laser systems is assumed.

\section{SC301 - Quantum Cascade Lasers: Science, Technology, Applications and Markets \\ Sunday, 05 June, 13:30-17:30 \\ Federico Capasso, Harvard Univ., USA}

Short Course Level: Beginner

Short Course Description: Quantum Cascade Lasers (QCLs) are fundamentally different from diode lasers due to their physical operating principle, which makes it possible to design and tune their wavelength over a wide range by simple tailoring of active region layer thicknesses, and due to their unipolar nature. Yet they use the same technology platform as conventional semiconductor lasers. These features have revolutionized applications (spectroscopy, sensing, etc.) in the mid-infrared region of the spectrum, where molecules have their absorption fingerprints, and in the far-infrared or so called Terahertz spectrum. In these regions until the advent of QCLs there were no semiconductor lasers capable of room temperature operation in pulsed or cw, as well high output power and stable/wide single mode tunability. The unipolar nature of $\mathrm{QCL}$, combined with the capabilities of quantum engineering, leads to unprecedented design flexibility and functionality compared to other lasers. The physics of QCLs, design principles, supported by modeling, will be discussed along with the electronic, optical and thermal properties. State-of-the-art performance in the mid-ir and Terahertz will be reviewed. In particular high power CW room temperature
QCLs, broadly tunable QCL, short wavelength MWIR QCLs and recent breakthroughs in $\mathrm{THz}$ room temperature operation will be presented. A broad range of applications (IR countermeasures, stand-off detection, chembio sensing, trace gas analysis, industrial process control, medical and combustion diagnostics, imaging, etc.) and their ongoing commercial development will be discussed.

\section{Short Course Benefits:}

This course should enable you to:

- Describe underlying QC Laser physics, operating principles and fundamental differences between standard semiconductor lasers and QC lasers

- Explain quantum design of the key types of QC lasers, which have entered real world applications, and how their electrical and optical properties can be tailored to optimize performance in the mid-infrared and $\mathrm{THz}$ regions

- Discuss experimental device performance, including physical limits, design constraints and comparison with theory and determine device characteristics (currentvoltage and light-current curves; differential and power efficiency, threshold, gain and losses; spectral behavior, single mode operation; high speed operation)

- Explain the basics of QC laser device technology: fabrication process, materials growth options

- Illustrate the basics of a chemical sensing system; discuss applications of state-of the-art mid-infrared $\mathrm{OC}$ lasers to sensing and present several examples of $\mathrm{QC}$ laser commercialization

- Discuss current and future markets of QC lasers

Short Course Audience: This course is for graduate students; qualified undergraduates (mostly senior level) majoring in EE or physics/applied physics; researchers in industry, academia and government labs; engineers, sales reps and technical managers.

\section{SC378 - Introduction to Ultrafast Optics}

Sunday, 05 June, 13:30-17:30

Rick Trebino, Georgia Institute of Technology, USA

\section{Short Course Level: Beginner}

Short Course Description: Ultrafast Optics-the science and technology of ultrashort laser pulses-is one of the most exciting and dynamic fields of science. While ultrashort laser pulses seem quite exotic (they're the shortest events ever created!), their applications are many, ranging from the study of ultrafast fundamental events to telecommunications to micromachining to biomedical imaging - to name a few. Interestingly, these lasers are readily available, and they are easy to understand. But their use requires some sophistication. This course is a basic introduction to the nature of these lasers and the pulses they generate. It will discuss the principles of their generation and amplification and describe their most common distortions in space and time and how to avoid them-or take advantage of them. In addition, it will cover the nonlinear optics of ultrashort pulses for converting pulses to almost any color, as well as the additional interesting and potentially deleterious effects nonlinear optical processes can cause. Finally, it will cover techniques for ultrashort-pulse measurement. 


\section{Short Course Benefits:}

This course should enable you to:

- Explain how ultrashort-pulse lasers and amplifiers work.

- Describe and describe ultrashort pulses and their many distortions.

- Use nonlinear optics to an convert ultrashort laser pulse to virtually any wavelength.

- Take advantage of-or avoid—nonlinear-optical highintensity effects.

- Meaningfully measure ultrashort pulses.

Short Course Audience: Any scientist or engineer interested in the science and technology of the shortest events ever created, especially those new to it.

\section{SC396 - Frontiers of Guided Wave Nonlinear Optics Sunday, 05 June, 13:30-17:30 \\ Ben Eggleton, Univ. of Sydney, Australia}

\section{Short Course Level: Advanced Beginner}

Short Course Description: This course will review recent research and applications in the field of nonlinear guided wave optics with emphasis on both fundamentals and emerging applications. Starting from a strong foundation in the principles of nonlinear optics, we will review recent progress in emerging nonlinear optical platforms with an emphasis on the different materials, including silicon, chalcogenide, III-V semiconductors, lithium niobate, photonic crystal fibres, nanophotonic circuits and others. We will establish key figures of merit for these different material systems and a general framework for nonlinear guided wave optics with emphasis on the applications in emerging areas of science and technology. We will then review recent progress and breakthroughs in the following areas: All-optical processing, Ultra-fast optical communications, Slow light, highly nonlinear and emerging waveguides, Ultrafast measurement and pulse characterization, Frequency combs and optical clock, Optical parametric amplifiers and oscillators, Generation and applications of optical super-continuum, Nonlinear localization effects and solitons, Nonlinear optics for quantum information.

\section{Short Course Benefits:}

This course should enable you to:

- Discuss state of the art knowledge of nonlinear optics in emerging waveguides and materials

- Explain the applications of nonlinear optics in key applications

- Describe the foundation of nonlinear waveguide physics for emerging applications and science

Short Course Audience: This course assumes some basic knowledge/familiarity of nonlinear optics. Individuals lacking such knowledge should consider taking SC149: Foundations of Nonlinear Optics first.
SC362 - Cavity Optomechanics: Fundamentals and Applications of Controlling and Measuring Nanoand Micro-mechanical Oscillators with Laser Light Monday, 06 June, 09:00-12:00

Tobias Kippenberg, Ecole Polytechnique Federale de Lausanne, Switzerland

\section{Short Course Level: Advanced Beginner}

Short Course Description: Radiation pressure denotes the force that optical fields exert and which have wide ranging applications in both fundamental science and applications such as Laser cooling or optical tweezers. Radiation pressure can, however, also have a profound influence on micro- and nanophotonic devices, due to the fact that radiation pressure can couple optical and mechanical modes. This optomechanical coupling gives rise to a host of new phenomena and applications in force, displacement and mass sensing. This course is intended to give an introduction of the Physics and Applications of cavity optomechanics and highlight the rapid developments in this emerging field. Optomechanical coupling can be used to both cool and amplify mechanical motion and thereby allow new light driven photon clocks. Optomechanical refrigeration of mechanical modes gives insights into the quantum limits of mechanical motion. In addition, radiation pressure coupling enables new way of processing light all optically enabling optical mixers, delay lines or storage elements. Moreover, the basic limitations of optomechanical displacement measurements, due to quantum noise and practical laser phase noise limitations, will be reviewed, relevant across a wide range of sensing experiments.

The course will make contact to practical applications of optomechanics in Metrology (force sensors, mass sensors and light driven optical clocks) and review fundamental design principles of optomechanical coupling and the design of high $\mathrm{Q}$ mechanical oscillators. The use of finite element simulations will be covered.

\section{Short Course Benefits:}

This course should enable you to:

- Explain gradient and scattering light forces in microcavities and micromechanical systems

- Design high -Q nano-and micro- mechanical oscillators (finite element modeling, FEM)

- Discuss the fundamental limits of mechanical $\mathrm{Q}$ in NEMS/MEMS

- Describe of the fundamental and practical limits of displacement sensors

- Summarize Applications of optomechanics in mass and force sensing

- Explain the basic optomechanical phenomena (amplification, cooling)

- Discuss the standard quantum limit (SQL)

- Characterize radiation pressure driven oscillations in terms of fundamental oscillator metrics

- Define Phase and frequency noise of oscillators 
- Know the influence of phase and amplitude noise of a wide variety of laser systems (fiber lasers, TiSa, diode lasers) in optomechanical systems

Short Course Audience: This course is intended for physicists and optical and electrical engineers desiring both focused fundamental knowledge of cavity optomechanical coupling (i.e., radiation pressure coupling of light and NEMS/MEMS) but also a view of emerging applications of this new technology. The instruction will be at a level appropriate for graduate students and will assume some basic knowledge of laser.

\section{SC376 - Plasmonics}

Monday, 06 June, 09:00-12:00

Mark Brongersma, Stanford Univ., USA

Short Course Level: Beginner

Short Course Description: Plasmonics is an exciting new field of science and technology that aims to exploit the unique optical properties of metallic nanostructures to enable routing and active manipulation of light at the nanoscale. Nanometallic objects derive these properties from their ability to support collective electron excitations, known as surface plasmons (SPs). Presently we are witnessing an explosive growth in both the number and range of plasmonics applications; it is becoming eminently clear that both new fundamental science and device technologies are being enabled by the current plasmonics revolution. The intention of this course is to give the participants a fundamental background and working knowledge of the main physical ideas used in plasmonics, as well as an overview of modern trends in research and applications.

The Short Course will begin with a general overview of the field of plasmonics. This will be followed by an introduction to the basic concepts that enable one to understand and design a range of plasmonic functionalities. This part will be followed by an in-depth discussion of a range of active and passive plasmonic devices that have recently emerged. Particular attention will be given to nanometallic structures in which surface plasmons can be generated, routed, switched, amplified, and detected. It will be shown that the intrinsically small size of plasmonic devices directly results in higher operating speeds and facilitates an improved synergy between optical and electronic components. The field of plasmonics is rapidly growing and has started to provide a whole range of exciting new research and development opportunities that go well beyond chipscale components. A number of such developments will be investigated, including new types of optical sensors, solar cells, quantum plasmonic components, non-linear, and ultrafast devices. At the end of the tutorial, a critical assessment of the entire field is given and some of the truly exciting new opportunities for plasmonics are identified. A comparison of metallic and high-index semiconductor antennas and metamaterials will be made as well.

\section{Short Course Benefits:}

This course should enable you to:

- Identify key physical concepts used in Plasmonics that enable light manipulation at ultra small length- and time-scales.

- Explain choices of different metal types, shapes, and sizes to accomplish different plasmonic functionalities.
- Summarize common electromagnetic computational tools to design plasmonic structures and devices.

- Discuss the current state of the field in terms of fundamental understanding as well as device applications.

- Discuss the most recent trends and developments in research and applications.

Short Course Audience: Optical engineers and scientists who are interested in learning about the rapidly emerging field of plasmonics and its potential impact.

\section{SC440 - How to Communicate High-Tech to the Market - A Marketing and PR \\ Monday, 06 June, 09:00-12:00 \\ Andreas Thoss, THOSS Media GmbH, Germany}

Short Course Level: Intermediate

Short Course Description: Winning customers for a new high-tech product is far from easy. But it has been done many times. This workshop gives hands-on advice for the communication of new technologies to prospective markets.

Attendees will learn how to set up an efficient integrated communication strategy for a given technology or product and what they have to do to implement this strategy and to meet the respective targets.

\section{Short Course Benefits:}

This course should enable you to:

- Identify target audiences

- Identify proper means to address these target audiences efficiently

- Set up a text toolbox

- Select the right media channels for effective communication

- Set up a detailed communication plan for the next 12 months

Short Course Audience: This course is intended for scientists \& engineers with interest in technology transfer and potential high-tech startup founders. Also Public Relations staff from scientific institutions and the high-tech industry.

\section{SC424 - Optical Terahertz Science and Technology} Monday, 06 June, 12:30-15:30

David G. Cooke; Dept. of Physics, McGill University, Canada

\section{Short Course Level: Advanced Beginner}

Short Course Description: The purpose of this short course is to introduce time-domain optical techniques based on femtosecond lasers for generating, manipulating and detecting light in the $0.1-10 \mathrm{THz}$ region, and demonstrate how this interesting part of the spectrum can be used to improve our understanding of materials. I will discuss THz imaging and sensing applications that are driving the development of this technology and discuss new physics that can be probed with short pulses of $\mathrm{THz}$ light. 
Course outline:

- The THz spectrum

- Optical methods for THz generation

- Techniques for $\mathrm{THz}$ detection

- Optics for THz light

- THz waveguides

- THz imaging

- THz time-domain spectroscopy

- Applications of THz light

Short Course Benefits:

This course should enable you to

- Explain methods for the generation and detection of coherent terahertz radiation

- Explain and apply methods for terahertz time - domain spectroscopy

- Understand the physical phenomena taking place on the picosecond - time scale

- Gain hands-on experience with advanced experimental equipment and numerical tools

- Understand and explain the pump - probe techniques for time-resolved spectroscopic measurements in the terahertz range

- Explain and interpret experimental measurements based on the theoretical models

Short Course Audience: This course is aimed at graduate students interested in the field of time-domain $\mathrm{THz}$ spectroscopy and imaging techniques. Basic knowledge of electromagnetic waves and condensed matter systems is suggested.

\section{SC439 - Attosecond Optics NEW}

Monday, 06 June, 12:30-15:30

Zenghu Chang, Univ. of Central Florida, USA

\section{Short Course Level: Beginner}

Short Course Description: Since the invention of lasers in 1960, various techniques such as mode-locking have been developed to push the pulse duration down first to picoseconds and then to femtoseconds, which is the oscillation period of infrared and visible light. The generation of attosecond pulses requires new methods to produce broadband coherent electromagnetic waves in the UV to $x$-ray range because of the lack of proper gain media. The discovery of high-order harmonic generation in high intensity laser-atom interaction at the end of 1980s paved the way. In 2001, attosecond light pulses, a train of attosecond bursts or single isolated attosecond pulses, were measured for the first time. It was accomplished by first converting the attosecond photons to photoelectrons in a combination of weak extreme ultraviolet and strong infrared fields, and then retrieve the spectrum phase of the attosecond pulse by reconstructing the photoelectron spectrum. Since then, various sub-optical-cycle gating schemes such as polarization gating and Double Optical Gating have been demonstrated to generation isolated attosecond pulses. By properly compensating the intrinsic chirp, 67 as pulses were characterized in 2012, which is so far the shorted light pulses. The new frontier in attosecond optics research is to significantly increase the photon flux and to extend the spectrum to the "water window." This course covers High harmonic generation, Carrier-envelope phase of femtosecond driving lasers, Semi-classical model and Strong Field Approximation, Phase-matching in partially ionized media, Sub-cycle gating and attosecond pulse characterization, Attosecond streaking and transient absorption spectroscopy.

\section{Short Course Benefits:}

This course should enable you to:

- Specify parameters of femtosecond driving lasers that are critical to the generation of attosecond pulse trains and single isolated attosecond pulses

- Compare pros and cons of driving lasers based on Ti:Sapphire Chirped Pulse Amplification and Optical Parametric Amplifiers

- Explain the principle and techniques of locking the carrier-envelope offset frequency of femtosecond oscillators and carrier-envelope phase of amplified pulses

- Define short and long trajectories in the attosecond generation process using the Strong Field Approximation in the Lewenstein model

- Estimate the cutoff photon energy and attosecond chirp using the semi-classical model

- Calculate ionization probability of atoms in an intense laser field with the Ammosov-Delone-Krainov (ADK) tunneling rate

- Describe the principle of attosecond streak camera for characterizing attosecond pulses

- Identify the major factors that affects the phase matching of high harmonic generation in partially ionized media.

Short Course Audience: This short course targets senior undergraduate students, graduate students, postdoc fellows, scientists and engineers seeking to enter attosecond optics. The audience should have studied electromagnetism, optics, lasers, quantum mechanics and atomic physics at undergraduate or graduate levels. Prior knowledge of femtosecond lasers is required. Although basic theory is covered, it emphasizes on experimental aspects of attosecond optics, such as locking the carrier-envelope phase of the driving lasers and designing time-of-flight spectrometers for attosecond streak cameras.

\section{SC352 - Introduction to Ultrafast Pulse Shaping-- Principles and Applications \\ Tuesday, 07 June, 12:00-15:00 \\ Marcos Dantus, Michigan State Univ., USA}

\section{Short Course Level: Advanced Beginner}

Short Course Description: This course begins by describing pulse shaping with a hands-on computer simulation that allows one to get a sense of how femtosecond pulses change in response to different phases and amplitudes. The essential physics and a brief background of the development of shapers are provided. The course goes over the experimental implementation requirements and then covers some of the most salient applications of pulse shapers, among them are 
pulse compression pulse characterization, creation of two or more pulse replicas, control of nonlinear optical processes such as selective two-photon excitation and selective vibrational mode excitation, material processing, microscopy and others.

\section{Short Course Benefits:}

This course should enable you to:

- Gain a better understanding of femtosecond laser pulses and their applications

- Learn pulse shaper design principles

- Compare among different pulse shaper designs and to determine which one is best suited for a particular application

- Simulate the output pulse from a pulse shaper given a particular phase and amplitude modulation

- Predict the effect caused by introducing a simple phase such as a linear, quadratic or cubic function on a transform-limited pulse

- Learn two different approaches to creating pulse replica that can be independently controlled with attosecond precision in the time domain using the pulse shaper

- Measure the spectral phase of laser pulses using the pulse shaper itself as the measurement tool, and eliminating phase distortions to compress the output pulses

- Summarize the advantages of having an adaptive pulse shaper for controlling the output of ultrafast lasers

Short Course Audience: This course, updated yearly, is intended for everyone that uses or intends to use femtosecond laser pulses in academic research or industry. Attendees will learn how pulse shaping can greatly enhance femtosecond laser applications. No prior knowledge about pulse shaping is required.

\section{SC410 - Finite Element Modeling Methods for Photonics and Optics}

Tuesday, 07 June, 12:00-15:00

Arti Agrawal, City Univ., UK

\section{Short Course Level: Beginner}

Short Course Description: Numerical modelling and simulation of optical devices and components is a key tool in improving performance by reducing time and monetary costs, design optimization and characterization as well as innovating new ideas. Both passive and active devices are modelled and optimized numerically. In some cases simulation is the only way to explore phenomena where technology is not advanced enough for fabrication. The interaction of the optical beam with physical effects such as non-linearity, stress, strain, change in refractive index due to temperature, application of electric fields etc. are now extremely important. Modelling complements experimental work perfectly and almost no research is conducted without it.

The Finite Element (FE) method is one of the most popular and powerful methods for modelling in Photonics. This short course starts with Maxwell's equations and explains the basic principles of numerical modelling and the key assumptions involved. This foundation is used to develop the FE method, including a brief tour of the mathematics. How the method can be applied to various optical devices is discussed in detail. How can physical effects be included with the FE method for modelling is considered. The course ends with an explanation of FE based beam propagation methods and how these can be used to find the evolution of the optical fields.

Methods covered include full vector Finite Element method for modal solutions; FEM with physical effects; and Finite Element Beam Propagation Methods (FE BPM). Practical illustrations include optical fibers including photonic crystal fibers; Si slot waveguides, nanowires and high index contrast structures; bent waveguides and loss; plasmonic waveguides; and Second Harmonic generation in waveguides: FE BPM.

\section{Short Course Benefits:}

This course should enable you to:

- Identify and explain basic principles of numerical modelling in Photonics

- Discuss and explain Full vector Finite Element Method (FEM) for modal solutions

- Discuss FEM with physical effects (non-linearity, stress/ strain, acousto-optic, electro-optic effect etc.)

- Discuss Finite Element Beam Propagation Methods (FE BPM)

- Discuss and explain how to incorporate Perfectly Matched Layer and Periodic boundary condition

- Summarize how to generate mesh for structures and post-processing of results

- Tips on how to best utilise commercial software

- Discuss the application of the method to practical devices: nano wires, optical fibers, sensors etc.

- Identify the appropriate modeling method for their problem

- How to incorporate PML boundary conditions and write your own code

Short Course Audience: This course is intended for researchers, engineers and students who use simulation in their work in both fundamental and applied aspects of Optics and Photonics, especially for components and devices. The course is useful for members of both academic and industrial institutions. Basic background and familiarity in Optics will be sufficient.

\section{SC270 - High Power Fiber Lasers and Amplifiers} Tuesday, 07 June, 12:00-16:00

W. Andrew Clarkson, Optoelectronics Res. Ctr., Univ. of Southampton, UK

\section{Short Course Level: Advanced Beginner}

Short Course Description: Recent advances in claddingpumped fiber lasers and amplifiers have been dramatic, leading to unprecedented levels of performance in terms of output power, efficiency, beam quality and wavelength coverage. These achievements have attracted growing interest within the community and have fueled thoughts that fiber-based sources may one day replace conventional "bulk" solid-state lasers in many application areas. The main attractions of cladding-pumped fiber sources are derived directly from their 
geometry, which simultaneously allows very efficient generation of coherent light and almost complete immunity from the effects of heat generation, which are so detrimental to the performance of other types of lasers.

This course aims to provide an introduction to high power fiber lasers and amplifiers, starting from the basic principles of operation and ending with examples of current stateof-the-art devices and some thoughts on future prospects. The course will cover a range of topics, including basic fiber laser and amplifier theory, spectroscopy of the relevant rare earth ions for high power devices, a discussion of the factors influencing laser and amplifier performance, fiber design and fabrication, pump sources and pump launching schemes, fiber resonator design, master-oscillator and power-amplifier configurations, linewidth control and wavelength selection, transverse mode selection, nonlinear loss processes (SBS and SRS) and their impact on performance, and heat generation and its impact on power scalability. The course will also give an overview of techniques (e.g. coherent and spectral beam combining) for further scaling of output power and provide an introduction to hybrid fiber-bulk laser schemes for scaling pulse energy.

\section{Short Course Benefits:}

This course should enable you to:

- Calculate threshold pump power and slope efficiency, and estimate the maximum output power that can be obtained from a given fiber laser oscillator or amplifier configuration

- Select the optimum pump source for a given rare earth ion transition and fiber design

- Design the pump light collection and coupling scheme and estimate the pump launch efficiency

- Specify the fiber parameters (e.g., cladding design, core size, rare earth ion concentration) required for a particular laser or amplifier configuration

- Design the fiber laser resonator and amplifier and select the operating wavelength

- Estimate thermally induced damage limit

- Estimate the power scaling limit

- Measure fiber laser performance characteristics and relate these to fiber design and resonator parameters

Short Course Audience: This course is intended for individuals with a basic knowledge of lasers and optics who wish to learn about the basic principles and capabilities of fiber lasers and amplifiers when operating at high power levels. The course will also cover some of the practical issues of operating these devices and provide an update for those wishing to learn about some of the latest developments in this rapidly advancing field.

\section{SC438 - Photonic Metamaterials NEW}

Tuesday, 07 June, 12:00-16:00

Nader Engheta, University of Pennsylvania, USA

Short Course Level: Beginner

Short Course Description: Controlling electromagnetic and optical fields and waves can be achieved via materials. The wave-matter interaction can be engineered using structures made of materials with required parameters and structures with selected shapes, dimensions and sizes. Recent advances in materials science and engineering, condensed matter physics, optical materials, nanoscience and nanotechnology have made it possible to tailor materials with unusual parameters and characteristics. The field of metamaterials, along with its two-dimensional version known as metasurfaces, has seen growing interest and extensive development in recent years. Metamaterials are engineered composite structures made of subwavelength inclusions with suitable materials and proper arrangements. The compositions, arrangements, alignments, densities and distributions of these building blocks in host media provide a variety of degrees of freedom in the design of light-matter interaction with such structures. Manipulation of light at the nano-, micro-, meso- and macroscales using metamaterials and metasurfaces provides rich platforms for tailoring electromagnetic waves with desired functionalities.

In this tutorial, we will begin with the basics of electromagnetic wave interaction with material media and structures. Then the course will get into some of the specifics of the characteristics of metamaterials and metasurfaces including the dispersion properties, scattering mechanisms, effectivemedium phenomena, and unconventional features of waves in such environments. We will then discuss some of the specific topics in photonic metamaterials such as extreme-parameter metamaterials (i.e., epsilon-near-zero (ENZ), mu-near-zero (MNZ), and epsilon-and-mu-near-zero (EMNZ) structures) and their specialized wave-matter interactions, graphene metamaterials as a platform for ideas for one-atom-thick optical device concepts, optical metatronics ("lumped" nanocircuitry) and informatic metastructures for photonic information processing and computing at the nanoscale, scattering engineering using metamaterials (such as cloaking), guided waves in metamaterials, and nonreciprocal metastructures. Various features and potential applications of these topics will also be presented and discussed. During the course, we will have interactive discussions and question-answer sessions.

\section{Short Course Benefits:}

This course should enable you to:

- Describe the basics of electromagnetic field and wave interaction with metamaterials and metasurfaces

- Explain some of the important properties of photonic metamaterials

- Discuss some of the scenarios in light-matter interaction with "extreme-parameter" metamaterials

- Describe the fundamentals of optical nanocircuits ("optical metatronics"), with potentials for information processing in nanophotonics

- Explain some of the salient features of scattering and guidance of lights in metamaterials and metasurfaces

Short Course Audience: Graduate students and senior undergraduates with EE, Physics, and Applied Physics interests; Engineers, researchers and technical managers from industry, government labs, and universities; Introductory knowledge of electromagnetics and optics is required. 


\section{Special Events}

\section{Cheeky Scientist Workshops \& Reception \\ Sunday, 5 June, 13:00-18:00}

Marriott Salon III and Foyer

Workshops are complimentary for OSA Members. There is limited space at each. RSVP online at cleoconference.org/ cheekyscientist

Would you like a referral to a job that pays $\$ 100 \mathrm{k}$ with benefits? Here's a better question, if I gave you a referral to that job, would you know what to do with it? Would you be able to confidently carry that referral through to securing an interview and negotiating your salary to $\$ 110 \mathrm{k}$ with benefits and relocation benefits? Isaiah Hankel works with hundreds of graduate students and postdocs daily helping them to transition to industry by first showing them how to present themselves as business professionals. Learn the credibility indicators that hiring managers and recruiters look for when choosing a candidate to bring in for an interview and how to implement them into every aspect of your transition.

Workshop Schedule

The Tailored Job Search: 13:00-14:30

From Online to In-person: the Job-seekers Guide to Networking: 15:00-16:30

Cheeky Scientist Workshop Networking Reception: 16:30-18:00

Hosted by:
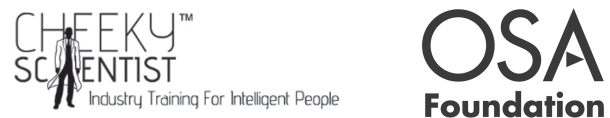

\section{OSA Workshop on New Regimes for} Nonlinear Optics

Monday, 6 June, 12:30-13:30

Room 231B

\section{Sponsored by}

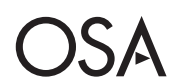

Short Wavelength Sources and Attosecond/High Field Physics Technical Group

Join the OSA Short Wavelength Sources \& Attosecond/High Field Physics Technical Group for a special workshop featuring talks on attosecond nonlinear optics and facilitating collaboration between researchers in these areas. Includes lunch; RSVP required. Contact TGactivities@osa.org to register, pending availability.

\section{OSA Technical Group Poster Session \\ Monday, 6 June, 19:00-20:00 \\ Room 230B}

\section{Sponsored by OSA Technical Groups}

Join the OSA Technical Groups for a series of focused poster sessions. These sessions will bring together students and colleagues for an opportunity to share their latest research findings, exchange ideas, and facilitate collaborations in relevant areas. Participating technical groups include:

- Optical Biosensors Technical Group

- Optical Material Studies Technical Group

- Optoelectronics Technical Group

- Nanophotonics Technical Group

\section{Pizza Lunch at the CLEO:EXPO}

Tuesday, 7 June, 11:30-13:30

Exhibit Halls 1, 2 \& 3

Kick-off the CLEO:Expo opening and join your colleagues on the CLEO show floor to enjoy a pizza lunch and the first networking opportunity with the exhibitors.

\section{VIP Industry Leaders Networking Event: Connecting Corporate Executives, Young Professionals and Students \\ Tuesday, 7 June, 12:00-13:30 \\ Exhibit Hall 1}

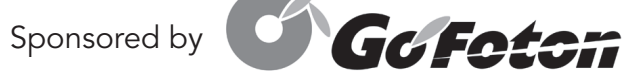

This session brings together Industry Executives to share their business experience - from how they started their careers and lessons learned along the way, to using their degree in an executive position - with Young Professionals and Students. The program starts with informal networking during lunch and then transitions into "speed meetings" - small, brief visits with each executive to discuss careers, industry trends or other career topics. This program is free of charge and includes a box lunch for students and young professionals.

Advance registration is required. Contact vipevents@osa.org to register for this event.

\section{Meet the OSA Editors' Reception \\ Tuesday, 7 June, 17:00-18:30 \\ Market Terrace}

Join OSA Publishing's Journal Editors for conversation and refreshments. The Editors welcome your questions, concerns and ideas for any of OSA's Journals. Topics that can be covered include best practices when submitting a manuscript; elements of a useful manuscript review; criteria editors look for in submitted manuscripts; and the process to propose a Feature Issue topic for publication in an OSA Journal. All are welcome.

\section{Conference Reception and Poster Session}

Tuesday, 7 June, 18:00-19:30

Exhibit Halls 1, 2 \& 3

Sponsored by Th:

Enjoy a festive evening with your colleagues, while intermingling with the exhibitors and viewing the first poster session. Full conference technical attendees will receive drink tickets with their registration material and a cash bar will also be available to purchase drinks. The reception is open to all attendees and badges must be worn to enter the reception.

All conference locations are in the San Jose Convention Center unless otherwise noted. 


\section{Poster Sessions}

Exhibit Halls 1, 2 \& 3

Poster Sessions are an integral part of the Technical program. Each author is provided with a board with six-foot-high by four-foot-wide $(183 \mathrm{~cm} \times 128 \mathrm{~cm})$ of usable space on which to display the summary and results of his or her paper. Authors should remain in the vicinity of their presentation board for the duration of the sessions to answer questions from attendees. Authors may set up one hour prior to their assigned session and must remove their poster one hour following the session. Authors may submit their poster PDF to cstech@osa. org for publication.

\begin{tabular}{|l|l|}
\hline Tuesday, 7 June & $18: 00-19: 30$ \\
\hline Wednesday, 8 June & $10: 00-12: 00$ \\
\hline Thursday, 9 June & $11: 30-13: 00$ \\
\hline
\end{tabular}

\section{OSA Members, Family and Friends Event}

Wednesday, 8 June, 9:30-12:00

OSA members and their families are welcome to attend a 90-minute tour of the Moffett Field Historical Society Museum, which is dedicated to the education and preservation of one of northern California's most significant historic sites. Moffett Field has a storied and important history, which includes major contributions to U.S. national security and the advancement of science and technology. Named after Rear Admiral William Moffett, the museum includes exhibits on the U.S. Army Air Corps, WWII fighter planes, and NASA's experimental flight testing, wind tunnel experiments, and space flight simulation.

Shuttle transportation will depart from the Hilton Hotel on Almaden Boulevard at 9:30 and return at 12:00.

Registration is required. Please visit the OSA Membership booth to reserve your space.

\section{Climbing the ladder: Insights from leaders in Photonics Panel Discussion and Networking Lunch}

Wednesday, 8 June, 11:00-13:00

Almaden Ballroom, Hilton San Jose

The panel will include leaders, varying in career stages and expertise, who will discuss their career paths, career challenges and experiences. The panel will explore and discuss their perspectives in regards to career growth and overall job satisfaction. In this respect, the panel will also spend some time to discuss specifically the importance of seeking mentors and advocates to help with career development.

Networking lunch will allow you to connect with a potential mentor or a mentee. We would like to encourage attendees interested in either role to be proactive during the event. We hope some of the attendees would walk out of the event with a successful connection. This event is sponsored by, Women in Photonics- Silicon Valley (WiP-SV), OSA Women in Optics and IEEE Photonics Women in Photonics.

Registration required and the event is currently sold out.
Happy Hour in Exhibit Hall

Wednesday, 8 June, 16:30-18:30

Sponsored by PHOTONICS) MEDIA)

Don't miss relaxing evening Happy Hour on the CLEO show floor. This will be a perfect time to relax after a full day of sessions and walking the show floor, while networking with exhibitors and colleagues. The happy hour is open to all attendees. Badges must be worn to enter the exhibit hall.

\section{OSA's Light the Future with Ray Kurzweil} Wednesday, 8 June, 18:30-20:00

Grand Ballroom

Join OSA as we celebrate our 100th anniversary with the Light The Future speaker series. Ray Kurzweil, inventor, author, futurist, will give a presentation on 'Business and Society in the Age of Accelerating Returns', moderated by Steven Chu, Nobel Laureate, former U.S. Secretary of Energy, OSA Fellow, OSA Honorary Member.

The 21 st century is an era in which the very nature of what it means to be human will be both enriched and challenged, as our species breaks the shackles of its genetic legacy, and achieves inconceivable heights of intelligence, material progress, and longevity. While the social and philosophical ramifications of these changes will be profound, and the threats they pose considerable, celebrated futurist Ray Kurzweil presents an inspiring vision of our ultimate destiny in which we will merge with our machines, can radically extend our lives, and are vastly more intelligent.

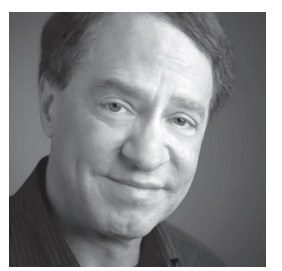

Ray Kurzweil has been described as "the ultimate thinking machine" by Forbes, and Inc. magazine ranked him \#8 among entrepreneurs in the United States, calling him the "rightful heir to Thomas Edison." $\mathrm{He}$ is considered one of the world's leading futurists, having predicted the age of mobile computing, digital books, wearables, self-driving cars and high-speed wireless data transmission. With a 30-year track record of accurate predictions, Kurzweil is currently heading up a team developing machine intelligence and natural language understanding as a Director of Engineering at Google.

\section{Snack Time}

Thursday, 9 June, 12:30-14:00

Exhibit Halls 1, 2, \& 3

Last chance to visit the CLEO: Expo! Grab a sweet treat as you network with exhibitors and see their innovative products and services that can help your organization.

\section{Postdeadline Paper Sessions}

Thursday, 9 June, 20:00-22:00

Locations announced on the Conference Update Sheet

The Technical Program Committee has accepted a limited number of postdeadline papers for oral presentation. The purpose of postdeadline papers is to give participants the opportunity to hear new and significant materials in rapidly advancing areas. 


\section{CLEO:EXPO}

Exhibit Halls 1, 2 and 3

Make sure to visit the exhibit floor, which features a diverse group of companies, representing every facet of the optics and photonics industries. Learn about new products, find technical and business solutions, and gain the most up-todate perspective of the laser-related business environment. Review the list of exhibitors below to see the wealth of companies you'll meet at CLEO:2016. Complete exhibition information is found in the Buyers' Guide.

There is no charge to attend the exhibit for conference registrants.

\begin{tabular}{|c|c|}
\hline Tuesday, 7 June & $11: 30-19: 30$ \\
Exhibit-Only Times & $11: 30-13: 30$ \\
& $15: 30-16: 00$ \\
& $18: 00-19: 30$ \\
\hline Wednesday, 8 June & $10: 00-18: 30$ \\
Exhibit-Only Times & $10: 00-13: 00$ \\
& $15: 00-15: 30$ \\
& $17: 30-18: 30$ \\
\hline Thursday, 9 June & $10: 00-15: 00$ \\
Exhibit-Only Times & $10: 00-14: 00$ \\
\hline
\end{tabular}

\section{Exhibit Hall Rules}

Children 12 and under must be accompanied by an adult at all times.

Strollers are not allowed on the show floor at any time.

Neither photography nor videotaping is permitted in the Exhibit Hall. Exhibitors need to get permission from Show Management to photograph their own booths. Non-compliance may result in the surrendering of film and removal from the hall.

For further questions, visit Registration on the Concourse Level.
Exhibitors (as of 30 March 2016)

ACT-Vision

Adamant Co., Ltd.

AdValue Photonics, Inc.

Advanced Thin Films

AdvR

Aerotech Inc.

AIP Publishing

Alnair Labs Corporation

Alpes Lasers SA

Alpine Research Optics

Altos Photonics, Inc.

American Physical Society (APS)

AMPHOS GmbH

Amplitude Systemes

Amplitude Technologies

APE - Applied Physics \& Electronics, Inc.

Asphericon

Atseva LLC

Attocube Systems, Inc.

AUREA Technology

BeamWise, Inc.

BioPhotonic Solutions Inc.

Boston Electronics Corporation

Brimrose Corporation of America

Bristol Instruments, Inc.

Calmar Laser, Inc.

CASTECH, Inc.

Changchun New Industries Optoelectronics Tech. Co. Ltd. Chroma Technology Corp.

Coherent, Inc.

CLEO:2016 Management thanks the following corporate sponsors for their generous support:

A AdValue Photonics

Pionium

(1) Nemport.

\section{PHYSICS TODAY}
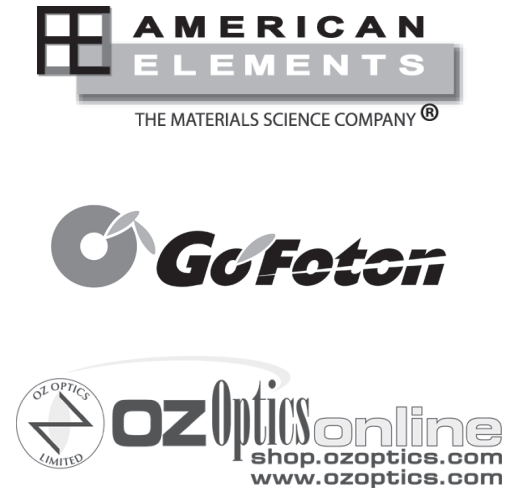

ThermoTek"
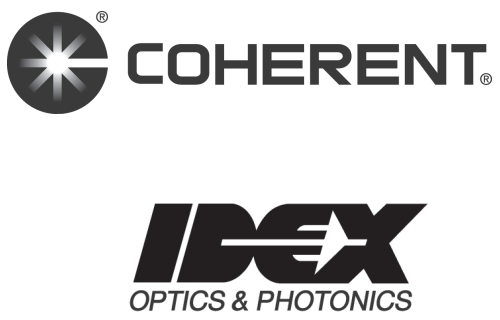

PHOTONICS) MEDIA

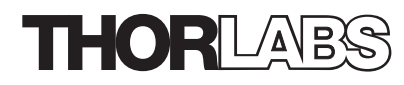

All conference locations are in the San Jose Convention Center unless otherwise noted. 
Continuum, Inc.

CorActive High-Tech, Inc.

Crestec Corporation

Crystalline Mirror Solutions, GmbH

CST of America, Inc.

CUNY, Advanced Science Research

Center

CVI Laser Optics

Cybel, LLC

DataRay, Inc.

Daylight Solutions, Inc.

DayOptics, Inc.

Discovery Semiconductors, Inc.

Edmund Optics, Inc.

EKSMA Optics

EKSPLA

Electro-Optics Technology, Inc.

Energetiq Technology, Inc.

EOSPACE, Inc.

\section{FASTLITE}

Femtochrome Research, Inc.

Fianium, Inc.

GAMDAN Optics

General Photonics Corp.

Gentec Electro-Optics, Inc.

Gooch \& Housego, PLC

GT Crystal Systems, LLC.

Hamamatsu Corporation

HC Photonics Corp.

Hiwin Corporation

HOLOEYE Photonics AG

Hortech Company

HTA Photomask

Ibsen Photonics A/S

ID Quantique

IDEX Optics \& Photonics

IDEX Optics \& Photonics Marketplace

IEEE Photonics Society

Imagine Optic, Inc.

IMRA America, Inc.

INNOLAS

Innovation Photonics

InPhenix

Inrad Optics

IRflex Corporation

Keopsys

KMLabs (Kapteyn-Murnane

Laboratories)

Kphotonics, LLC

Kyocera Industrial Ceramics

Corporation

Laser Focus World

Laser Quantum, Inc.
Lattice Electro Optics, Inc.

Light Conversion, Ltd.

Lighthouse Photonics

LightTrans $\mathrm{GmbH}$

Liquid Instruments

Luxmux Technology Corporation

M Squared Lasers Ltd.

Market Tech, Inc.

Menlo Systems

Mesa Photonics, LLC

Micro Photon Devices

Mindrum Precision, Inc.

MIRTHE Center - Princeton University

Montana Instruments

MONTFORT Laser GmbH

Nanjing University

National Energetics

Newport Corporation

NKT Photonics Inc.

Northrop Grumman Cutting Edge

Optronics, Inc.

Nufern

Nuphoton Technologies, Inc.

Ocean Optics, Inc.

OEwaves, Inc.

Optiforms

The Optronics Co., Ltd.

The Optical Society

OptiGrate

Optimax Systems, Inc.

OptoElectronic Components

OptoSigma Corporation

Optronis $\mathrm{GmbH}$

OSA 100 Exhibit

Osela, Inc.

Oxford University Press

Oxide Corporation

OZ Optics

PHASICS Corp.

Photodigm, Inc.

Photon Design

Photon Energy GmbH

Photonics Industries International

Photonics Media/Laurin Publishing

Photonix Edge, LLC

Photop Technologies, Inc

Physics Today

PI (Physik Instrumente) LP

PicoQuant Photonics North America, Inc.

Precision Glassblowing of Colorado

Princeton Instruments, Inc.

PriTel, Inc.
Pure Photonics

Quantum Design, Inc.

Quantum Opus

Radiantis

Raicol Crystals Ltd.

RESOLUTION spectra systems

RPMC Lasers, Inc.

Sacher Lasertechnik GmbH

Sandia National Laboratories

Santec USA Corporation

Seiwa Optical America, Inc.

Shasta Crystals

SILIOS TECHNOLOGIES

Siskiyou Corporation

SmarAct Inc.

Southern Photonics, Ltd.

Specialised Imaging

Spectrogon US, Inc.

Spectroscopy Magazine

SPIE: The Intl Society for Optics and Photonics

Springer

Stable Laser Systems

STANDA

Stanford Research Systems

StellarNet, Inc.

Swamp Optics, LLC

Synopsys

TelAztec

Teledyne Judson Technologies

ThermoTek, Inc.

Thorlabs

TMC Ametek

Toptica Photonics, Inc.

TRUMPF Inc.

University of Central Florida, CREOL

US Army Research Laboratory

Vescent Photonics, Inc.

Vigo Systems SA

Wiley

Wuhan Industrial Institute for Optoelectronics

Y\&M Technologies

Zaber Technologies

Zurich Instruments

All conference locations are in the San Jose Convention Center unless otherwise noted. 


\section{CLEO:EXPO Technology Playground}

Tuesday - Thursday, Exhibition Hours

Exhibit Hall

This interactive exhibits event allows you to network with top industry leaders, demo their innovative products/services and explore the latest innovations that can help you in your work. Visit all participating companies for a chance to win a $\$ 200$ American Express gift card. Stop by the OSA or IEEE Member booths for an entry form.

\section{Newport Student Lounge D Newport.}

All student attendees are invited to the Newport Student Lounge, co-sponsored by OSA. The lounge provides an opportunity to relax and spend time networking with other students, while enjoying complimentary, wireless Internet and refreshments.

\section{CLEO: Market Focus}

Tuesday, 7 June - Wednesday, 8 June

Exhibit Hall Theater

CLEO: Market Focus focuses on the latest trends in the photonics marketplace. The program provides a forum to discuss new products and emerging technologies and markets while also providing networking opportunities. All presentations and discussions will be focused on the latest in photonics products and services that have been playing an important role in the industry and those that potentially hold a future business opportunity. A key feature of this forum will be the survey of market trends and market sector outlook in the selected areas.

\section{Tuesday, 7 June}

13:00-14:30 Precision Manufacturing Using Ultrafast Lasers

15:30-17:00 AIM Photonics Update

\section{Wednesday, 8 June}

10:30-12:00 Update on the Optics and Photonics Industry and Forward-Looking Market Opportunities

15:00-16:30 Building the World's First Database of Matter: Spectroscopy in the Consumer's Hand

Visit www.cleoconference/marketfocus for complete information.

\section{Precision Manufacturing Using Ultrafast Lasers Tuesday, 7 June, 13:00-14:30}

Moderator: Mike Mielke, Lumentum, USA

Ultrafast lasers are optimum tools to machine, reshape, weld or otherwise modify a multitude of materials when required manufacturing precision approaches one micron. Conventional lasers focus energy in space and wavelength to achieve greater precision and cost-efficiency than mechanical tools or plasma discharge erosion. Ultrafast lasers add temporal focus of the energy to dramatically change the light-matter interaction. Typical benefits include enhanced dimensional accuracy and tighter tolerances, reduced collateral damage, and elimination of post-processing steps.
This panel will explore the trade-offs between using ultrafast lasers versus conventional tools for high volume, commercial product manufacturing. Topics will include the unique manufacturing precision enabled by ultrafast lasers, when this precision creates compelling economic advantage in the factory, how to identify the right type of laser source and workstation for your application, and the latest advancements in lasers and equipment. The session will consist of short presentations by industrial leaders in materials processing and conclude with a panel discussion open to audience questions.

\section{AIM Photonics Update \\ Tuesday, 7 June, 15:30-17:00 \\ Moderator: Todd Stievater, NRL, USA}

The American Institute for Manufacturing Integrated Photonics (AIM Photonics) was launched in 2015. This special panel session will bring together several members of the AIM Photonics leadership council to provide an update on the Institute and to answer questions from the audience.

\section{Update on the Optics and Photonics Industry and Forward-Looking Market Opportunities Wednesday, 8 June, 10:30-12:00}

Moderator: Tom Hausken, OIDA, USA

This session will review the current state of the optics and photonics industry with respect to revenues, product areas, and regions. There will also be a long view of the optics and photonics market opportunities across several sectors, drawing on some observations of our industry from the past, as well as promising markets going forward.

\section{Building the World's First Database of Matter: Spectroscopy in the Consumer's Hand A Special Enabled by Optics Session Wednesday, 8 June, 15:00-16:30}

Moderator: James Fisher, Newport, USA

Spectroscopy has been taking place in some form for more than 100 years, so what could be new at this point? One answer: scalability for consumer use. Enter SCiO, the first molecular sensor that fits in the palm of your hand. Through the science of spectroscopy, every time you scan with $\mathrm{SCiO}$, you're helping to build the world's first database of matter and opening a new world of discovery. For example, you can scan an apple, identify its unique properties, and upload its nutritional information into a tracking app or differentiate pharmaceuticals by chemical make-up. Speakers in this session will discuss the technology behind the award-winning $\mathrm{SCiO}$, the ability to scan a range of materials, and the user experience. And how does it really work? Attend this session and try it out yourself in a hands-on environment.

\section{Speakers:}

Damian Goldring, Consumer Physics, Israel

Yusuf Jamal, Analog Devices, Inc., USA

Gregory J. Quarles, The Optical Society, USA 
Thursday, 9 June, 10:00-13:00

Exhibit Hall Theater

Media Sponsor: PHOTONICS) MEDIA

The Technology Transfer Program provides a forum for entrepreneurs and researchers from startups, major universities, businesses, and national laboratories to present exciting new technologies that are ready and available for commercialization. The program starts with a keynote and is followed up with a tutorial for those who want to learn more about the licensing process_funding, entrepreneurship, technology transfer and intellectual property. During the Pitch Panel, attendees will hear from several organizations about their latest license-ready optics and photonics technologies (intellectual property from universities and laboratories) that could lead to new commercial products or improve the efficiency, durability or availability of existing components or systems. Organizations will also feature their license-ready technologies at tabletop displays in the exhibit hall.
10:00-10:30
10:30-11:30
Keynote
11:30-13:00
Tutorial: Technology Transfer
Pitch Panel

For complete information visit www.cleoconference.org/ techtransfer.

\section{Keynote Speaker}

Henry Kapteyn, KMLabs, USA

\section{Pitch Panel}

The Pitch Panel is an opportunity for entrepreneurs to showcase their technology, explain why its valuable and discuss the next steps to commercialization.

\section{Panelists:}

Magnus Bengtsson, Coherent, USA

Jason Eichenholz, Open Photonics, USA

Rick Plympton, Optimax, USA 


\section{CLEO Committees}

\section{CLEO: Applications \& Technology}

Nicusor Iftimia, Physical Sciences Inc., USA, General Chair Christian Wetzel, Rensselaer Polytechnic Inst., USA, General Chair

Peter E. Andersen, Danmarks Tekniske Universitet, Denmark, Program Chair

Dirk H. Sutter, TRUMPF Laser GmbH, Germany, Program Chair

\section{CLEO A\&T 1: Biomedical Applications}

Jin U. Kang, Johns Hopkins Univ., USA, Subcommittee Chair Chang-Seok Kim, Pusan National Univ., South Korea

Pilhan Kim, Korea Advanced Inst. Science \& Tech. (KAIST), South Korea

Xuan Liu, New Jersey Inst. of Tech., USA

Laleh Najafizadeh, Rutgers Univ., USA

Utkarsh Sharma, Carl Zeiss Meditec AG, USA

Melissa Skala, Vanderbilt Univ., USA

Benjamin J. Vakoc, Harvard Medical School, USA

Martin Villiger, Wellman Center for Photomedicine, USA

Peng Xi, Peking Univ., China

\section{CLEO A\&T 2: Industrial Applications}

Michael M. Mielke, Lumentum, USA, Subcommittee Chair Jiyeon Choi, Korea Inst. of Machinery \& Materials, South Korea

Peter Fendel, Thorlabs Inc, USA

Min Gu, Swinburne Univ. of Tech., Australia

Oleg Khodykin, KLA-TENCOR, USA

Jan Kleinert, ESI, USA

Xiang Peng, Raydiance Inc, USA

Jie Qiao, Rochester Inst. of Tech., USA

Jeffrey Wojtkiewicz, Nufern, USA

CLEO A\&T 3: Photonic Instrumentation \& Techniques for Metrology \& Industrial Process

Paul Williams, NIST, USA, Subcommittee Chair

Giorgio Brida, INRIM, Italy

Michael Frish, Physical Sciences Inc., USA

Jana Jágerská, UiT Norges Arktiske Universitet, Norway

Ekaterina A. Golovchenko, Tyco Electronics SubSea

Communications, USA

Erich N. Grossman, NIST, USA

Christian Pedersen, DTU Fotonik, Denmark

Andrew Sappey, Zolo Technologies, USA

\section{CLEO A\&T 4: Laser \& Photonics Applications for} Energy \& Environment

Stephanie Tomasulo, Naval Research Lab., USA, Subcommittee Chair

Kirstin Alberi, National Renewable Energy Lab., USA

David Bomse, Mesa Photonics, USA

Jordan Lang, Solar Junction, USA

Jan-Frederik Nekarda, Fraunhofer Inst. Solare Energie

Systeme, Germany

Joel Silver, Southwest Sciences Inc., USA

Homan Yuen, Solar Junction, USA

Mark A. Zondlo, Princeton Univ., USA

\section{CLEO: Science \& Innovations}

Seth Bank, Univ. of Texas at Austin, USA, General Chair

Valdas Pasiskevicius, Royal Inst. of Tech. (KTH), Sweden, General Chair

Nathan R. Newbury, NIST, USA, Program Chair

Jessie Rosenberg, IBM TJ Watson Research Center, USA, Program Chair

\section{CLEO S\&I 1: Light-Matter Interactions and Materials}

\section{Processing}

Emmanuel Haro-Poniatowski, UAM-Iztapalapa, Mexico, Subcommittee Chair

Feng Chen, Shandong Univ., China

Ya Cheng, Shanghai Inst. of Optics and Fine Mechanics, China

Maria Dinescu, NILPRP, Romania

Richard F. Haglund, Vanderbilt Univ., USA

Masaki Hashida, Kyoto Univ., Japan

Tsing-Hua Her, Univ. of North Carolina at Charlotte, USA

Alberto Pique, US Naval Research Lab., USA

Gagan Saini, Halliburton Energy Services, Inc., USA

Javier Solis, Instituto De Optica 'Daza De Valdes', Spain

CLEO S\&I 2: Advanced Science and Technology for Laser Systems and Facilities

Klaus Ertel, STFC Rutherford Appleton Lab, UK, Subcommittee Chair

Jake Bromage, Univ. of Rochester, USA

Gilles Cheriaux, Ecole Polytechnique ENSTA, France

Jay Doster, Northrop Grumman Cutting Edge Optronics, USA

Tae Moon Jeong, Gwangju Inst. of Science \& Tech., South Korea

Hiromitsu Kiriyama, Japan Atomic Energy Agency, Japan

Christian Kraenkel, Universität Hamburg, Germany

Thomas Metzger, TRUMPF Scientific Lasers GmbH, GERMANY

Brendan A. Reagan, Colorado State Univ., USA

Shawn Redmond, MIT Lincoln Lab, USA

Peter G. Schunemann, BAE Systems Inc., USA

Thomas Spinka, Lawrence Livermore National Lab, USA

\section{CLEO S\&I 3: Semiconductor Lasers}

Amr S. Helmy, Univ. of Toronto, Canada, Subcommittee Chair Mikhail A. Belkin, Univ. of Texas at Austin, USA

Maria Ana Cataluna, Univ. of Dundee, UK

Kent D. Choquette, Univ. of Illinois, USA

Ann Catrina Coleman, Univ of Texas at Dallas, USA

Raffaele Colombelli, Universite Paris Sud and CNRS, France

Sven Hoefling, Univ. of St Andrews, Germany

Fumio Koyama, Tokyo Inst. of Technology, Japan

Manabu Mitsuhara, NTT Basic Research Labs, Japan

Boon S. Ooi, King Abdullah Univ of Science \& Tech. (KAUST), Saudi Arabia

Michael C. Wanke, Sandia National Labs, USA

All conference locations are in the San Jose Convention Center unless otherwise noted. 
CLEO S\&I 4: Nonlinear Optical Technologies

Jeffrey Moses, Cornell Univ., USA, Subcommittee Chair

Masaki Asobe, Tokai Univ., Japan

Andrius Baltuska, Technische Universität Wien, Australia

Yen-Hung Chen, National Central Univ., Taiwan

Judith M. Dawes, Macquarie Univ., Australia

Leonel P. Gonzalez, Air Force Research Lab., USA

Nicolas Joly, Universität Erlangen-Nürnberg, Germany

Yoshitomo Okawachi, Columbia Univ., USA

Derryck T. Reid, Heriot-Watt Univ., UK

Michelle Sander, Boston Univ., USA

Jay E Sharping, Univ. of California Merced, USA

Irina T. Sorokina, Norges Teknisk Naturvitenskapelige Univ, Norway

Kartik Srinivasan, NIST, USA

\section{CLEO S\&I 5: Terahertz Science and Applications}

Dmitry Turchinovich, Max Planck Inst. for Polymer Research, Germany, Subcommittee Chair

Mona Jarrahi, Univ. of California Los Angeles, USA

Peter Uhd Jepsen, Danmarks Tekniske Universitet, Denmark

Hannah Joyce, Univ. of Cambridge, UK

Edmund Linfield, Univ. of Leeds, UK

Rajind Mendis, Rice Univ., USA

Hynek Nemec, Inst. of Physics of the Czech Academy of Sciences, Czech Republic

Marco Rahm, Technische Universität Kaiserslautern, Germany

Philip Taday, TeraView Ltd, UK

Masayoshi Tonouchi, Osaka Univ., Japan

Stephan Winnerl, Forschungszentrum Dresden-Rossendorf, Germany

\section{CLEO S\&I 6: Optical Materials, Fabrication and Characterization}

Amy C Foster, Johns Hopkins Univ., USA, Subcommittee Chair Juejun $\mathrm{Hu}$, MIT, USA

Ofer Levi, Univ. of Toronto, Canada

Michael Menard, UQAM., Canada

Thomas E. Murphy, Univ. of Maryland at College Park, USA

Jason Pelc, Hewlett Packard Labs, USA

Gregory Sun, Univ. of Massachusetts, USA

Takehiko Tawara, NTT Basic Research Labs, Japan

Fengqiu Wang, Nanjing Univ., China

Xi Xiao, FiberHome Technologies Group, China

\section{CLEO S\&I 7: Micro- and Nano-Photonic Devices}

Marcelo Davanco, NIST, USA, Subcommittee Chair Chin-Hui (Janet) Chen, Hewlett Packard Company, USA

Kenneth Brian Crozier, Univ. of Melbourne, Australia

Andrei Faraon, Caltech, USA

Alexander L. Gaeta, Cornell Univ., USA

Michael Galili, Danmarks Tekniske Universitet, Denmark

Wei Jiang, Rutgers Univ., USA

Christian Koos, Karlsruhe Inst. of Tech, Germany

Frank Koppens, ICFO, Spain

Qiang Lin, Univ. of Rochester, USA

Jeremy N. Munday, Univ. of Maryland at College Park, USA

Jon Schuller, Univ. of California Santa Barbara, USA

Takasumi Tanabe, Keio Univ., Japan

Hong Tang, Yale Univ., USA

Chee Wei Wong, Univ. of California Los Angeles, USA
CLEO S\&I 8: Ultrafast Optics, Optoelectronics \&

Applications

Takao Fuji, National Inst. of Natural Sciences, Japan, Subcommittee Chair

Andreas Assion, FemtoLasers Produktions GmbH, Australia Jose Azana, INRS-Energie Materiaux et Telecom, Canada

Ayhan Demircan, Leibniz Univ., Hannover, Germany

Christophe Dorrer, Univ. of Rochester, USA

Clemens Hoenninger, Amplitude Systemes, France

Jiro Itatani, ISSP, Univ. of Tokyo, Japan

Cristian Manzoni, IFN-CNR, Italy

Andrius Marcinkevicius, TRUMPF Inc., USA

Howard M. Milchberg, Univ. of Maryland at College Park, USA

Tenio Popmintchev, JILA, Univ. of Colorado at Boulder, USA

Thomas Sudmeyer, Université de Neuchâtel, Switzerland

Franz Tavella, SLAC National Accelerator Lab., USA

CLEO S\&I 9: Components, Integration, Interconnects and Signal Processing

Lin Zhu, Clemson Univ., USA, Subcommittee Chair

Adam Crook, Lockheed Martin, USA

Sasan Fathpour, Univ. of Central Florida (CREOL), USA

Mark Aaron Foster, Johns Hopkins Univ., USA

Qiaoqiang Gan, State Univ. of New York at Buffalo, USA

Hiroyuki Ishii, NTT Corporation, Japan

Leif Johansson, Freedom Photonics, LLC, USA

Mo Li, Univ. of Minnesota, USA

Marianna Pantouvaki, IMEC, Belgium

Stefan Francis Preble, Rochester Inst. of Tech., USA

Takahide Sakamoto, National Inst. of Information \& Comm. Tech., Japan

Weidong Zhou, Univ. of Texas at Arlington, USA

\section{CLEO S\&I 10: Biophotonics and Optofluidics}

Timo Mappes, Carl Zeiss Vison International GmbH, Germany, Subcommittee Chair

Dmitry V. Dylov, GE Global Research, USA

Ralph Jimenez, Univ. of Colorado at Boulder, USA

Ki Hean Kim, Pohang Univ of Science \& Technology, South Korea

Andreu Llobera, Centre Nacional de Microelectronica, Spain

Aydogan Ozcan, Univ. of California Los Angeles, USA

B. Hyle Park, Univ. of California Riverside, USA

Daniel Razansky, Helmholtz Zentrum München GmbH, Germany

Kevin K. Tsia, Univ. of Hong Kong, Hong Kong

\section{CLEO S\&I 11: Fiber Photonics}

Shinji Yamashita, Univ. of Tokyo, Japan, Subcommittee Chair Shahraam Afshar, Univ. of South Australia, Australia

Shaif-Ul Alam, Univ. of Southampton, UK

Fetah Benabid, Xlim Research Institute, France

Kin Seng Chiang, City Univ. of Hong Kong, Hong Kong

Andy Chong, Univ. of Dayton, USA

Liang Dong, Clemson Univ., USA

Peter D. Dragic, Univ. of Illinois at Urbana-Champaign, USA

Juliet Gopinath, Univ. of Colorado at Boulder, USA

Ming-lie Hu, Tianjin Univ., China

Khanh Quoc Kieu, Univ. of Arizona, USA

Jonathan C. Knight, Univ. of Bath, UK

Poul Kristensen, OFS Fitel Denmark I/S, Denmark

Sze Y. Set, Univ of Tokyo, Japan 


\section{CLEO S\&I 12: Lightwave Communications and Optical Networks}

Michael Vasilyev, Univ. of Texas at Arlington, USA, Subcommittee Chair

Anjali Agarwal, Applied Communication Sciences, USA Adolfo V. T. Cartaxo, Universidade de Lisboa, Portugal

Yue-Kai Huang, NEC Laboratories, USA

Nicolas K. Fontaine, Nokia Bell Labs, USA

David Jason Geisler, MIT Lincoln Lab, USA

Francesca Parmigiani, Univ. of Southampton, UK

Yikai Su, Shanghai Jiao Tong Univ., China

Takashi Sugihara, Mitsubishi Electric Corporation, Japan

Ioannis Tomkos, Athens Information Technology Center, Greece

\section{CLEO S\&I 13: Active Optical Sensing}

Mark Phillips, Pacific Northwest National Lab, USA, Subcommittee Chair

Adam J Fleisher, NIST, USA

Aleksandra Foltynowicz, Umea Universitet, Sweden

Scott Sheridan Howard, Univ. of Notre Dame, USA

R. Jason Jones, Univ. of Arizona, USA

Bernhard Lendl, Vienna Univ. of Technology, Austria Michal Nikodem, Wroclaw Research Centre EIT+, Poland

Christian Pflügl, Eos Photonics, Inc., USA

Dirk Richter, Univ. of Colorado, USA

Todd H. Stievater, US Naval Research Lab., USA

Damien Weidmann, STFC Rutherford Appleton Lab., UK Ian M. White, Univ. of Maryland at College Park, USA

Michael Wojcik, Space Dynamics Lab., USA

\section{CLEO S\&I 14: Optical Metrology}

Axel Ruehl, Deutsches Elektronen Synchrotron, Germany, Subcommittee Chair

lan R. Coddington, NIST, USA

Jerome Genest, Universite Laval, Canada

Jungwon Kim, Korea Advanced Inst. of Science \& Tech (KAIST), South Korea

Marco Andrea Marangoni, Politecnico di Milano, Italy

Michael J Martin, Sandia National Labs, USA

Mark Notcutt, Stable Laser Systems, USA

Franklyn Quinlan, NIST, USA

Stephane Schilt, Univ. of Neuchatel, Switzerland

Guanhao Wu, Tsinghua Univ., China

\section{CLEO: Fundamental Science}

Roberto Morandotti, INRS - Centre Énergie Matériaux Télécommunications, Canada, General Chair

William Munro, NTT Basic Research Laboratories, Japan, General Chair

Zhigang Chen, San Francisco State Univ., USA, Program Chair Jeffrey H. Shapiro, MIT, USA, Program Chair

\section{FS 1: Quantum Optics of Atoms, Molecules and Solids}

Sergey Polyakov, NIST, USA, Subcommittee Chair

Michal Bajcsy, Univ. of Waterloo, Canada

Daniel Felinto Pires Barbosa, Universidade Federal de Pernambuco, Brazil

Boris Blinov, Univ. of Washington, USA

Hugues de Riedmatten, ICFO -The Inst. of Photonic Sciences, Spain

Ivo Pietro Degiovanni, INRIM, Italy

Edward Flagg, West Virginia Univ., USA

Mohammad Hafezi, Joint Quantum Inst., USA
Andreas Muller, Univ. of South Florida, USA

Joshua Nunn, Univ. of Oxford, UK

Monika Schleier-Smith, Stanford Univ., USA

Olga V. Tikhonova, M. V. Lomonosov Moscow State Univ., Russia

Yanhong Xiao, Fudan Univ., China

Ite A. Yu, National Tsing Hua Univ., Taiwan

\section{FS 2: Quantum Science, Engineering and Technology}

Todd B. Pittman, Univ. of Maryland Baltimore County, USA, Subcommittee Chair

Konrad Banaszek, Uniwersytet Warszawski, Poland

Joshua Bienfang, NIST, USA

Warren P. Grice, Oak Ridge National Lab., USA

Elanor Huntington, Australian National Univ., Australia

Yoon-Ho Kim, Pohang Univ. of Science \& Tech., South Korea

Nathan Kim Langford, Technische Universiteit Delft, Netherlands

Olivier Pfister, Univ. of Virginia, USA

Qudsia Quraishi, US Army Research Lab., USA

Hiroki Takesue, NTT Basic Research Laboratories, Japan

Shigeki Takeuchi, Osaka Univ., Japan

Sebastien Tanzilli, Universite de Nice Sophia Antipolis, France

Tzu-Chieh Wei, State Univ of New York at Stony Brook, USA

\section{FS 3: Metamaterials and Complex Media}

Viktor Podolskiy, Univ. of Massachusetts Lowell, USA, Subcommittee Chair

Filippo Capolino, Univ. of California Irvine, USA

Nicholas Fang, MIT, USA

Dentcho Angelov Genov, Louisiana Tech Univ., USA

Zubin Jacob, Univ. of Alberta, Canada

Maria Kafesaki, Univ. of Crete, Greece

Stefan Linden, Universitat Bonn, Germany

Vinod M. Menon, City College of New York, USA

Alejandro Rodriguez, Princeton Univ., USA

Vera Smolyaninova, Towson Univ., USA

Alexey G Yamilov, Missouri Univ of Science \& Technology, USA

Lei Zhou, Fudan Univ., China

\section{FS 4: Optical Excitations and Ultrafast Phenomena} in Condensed Matter

Rohit Prasankumar, Los Alamos National Lab., USA, Subcommittee Chair

Hyunyong Choi, Yonsei Univ., South Korea

Keshav Moreshwar Dani, Okinawa Inst of Science \& Tech. Graduate Univ., Japan

Jeffrey Allan Davis, Swinburne Univ. of Tech., Australia

Kenan Gundogdu, North Carolina State Univ., USA

Matthias Clemens Hoffmann, SLAC National Accelerator Lab. USA

Chih-Wei Lai, Michigan State Univ., USA

Ermin Malic, Technische Universität Berlin, Germany

Richard Lunt Sandberg, Los Alamos National Lab., USA

Denis Seletskiy, Universitat Konstanz, Germany

Jigang Wang, lowa State Univ., USA

Haidan Wen, Argonne National Lab., USA

All conference locations are in the San Jose Convention Center unless otherwise noted. 


\section{FS 5: Nonlinear Optics and Novel Phenomena}

Stewart Aitchison, Univ. of Toronto, Canada, Subcommittee Chair

Matteo Clerici, Heriot-Watt Univ., UK

Xudong Fan, Univ. of Michigan, USA

Mercedeh Khajavikhan, Univ. of Central Florida (CREOL), USA

Zhiyuan Li, Chinese Academy of Sciences, China

Dragomir N. Neshev, Australian National Univ., Australia

Anna Claire Peacock, Univ. of Southampton, UK

Marco Peccianti, Univ. of Sussex, UK

Ulf Peschel, Universität Erlangen-Nürnberg, Germany

Luca Razzari, INRS-Energie Materiaux et Telecom, Canada

Venugopal Soma Rao, University of Hyderabad, India

Alexander Szameit, Friedrich-Schiller-Universität Jena, Germany

Frank W. Wise, Cornell Univ., USA

Jianke Yang, Univ. of Vermont, USA

\section{FS 6: Nano-Optics and Plasmonics}

Henri Lezec, NIST, USA, Subcommittee Chair

Amit Agrawal, NIST, USA

Javier Aizpurua, Material Physics Ctr. CSIC-UPV/ and DIPC, Spain

Jennifer Dionne, Stanford Univ., USA

Otto L Muskens, Univ. of Southampton, UK

Volker Jendrick Sorger, George Washington Univ., USA

Zheng Wang, Univ. of Texas at Austin, USA

Joel K. W. Yang, Singapore Univ. of Technology \& Design, Singapore

Xiaobo Yin, Univ. of Colorado Boulder, USA

Nanfang Yu, Columbia Univ., USA

Rashid Zia, Brown Univ., USA

\section{FS 7: High-Field Physics and Attoscience}

François Légaré, INRS- Énergie Matériaux Télécommunications Varennes, Canada, Subcommittee Chair

Michael Chini, Univ. of Central Florida, USA

Nirit Dudovich, Weizmann Inst. of Science, Israel

Matthias Fuchs, Univ. of Nebraska Lincoln, USA

Rodrigo Lopez-Martens, Laboratoire d'Optique Appliquée, France

Johan Mauritsson, Lund Univ., Sweden

Mauro Nisoli, Politecnico di Milano, Italy

Markus Roth, Technische Universität Darmstadt, Germany

Artem Rudenko, Univ. of Kansas, USA

Olga Smirnova, Max Born Inst., Germany

Eiji J. Takahashi, RIKEN, Japan

John W. G. Tisch, Imperial College London, UK

Donald P. Umstadter, Univ. of Nebraska Lincoln, USA

\section{CLEO Steering Committee}

\section{The Optical Society}

Timothy J. Carrig, Lockheed Martin Coherent Technologies, USA, Chair

Craig Arnold, Princeton Univ., USA

Ingmar Hartl, DESY, Germany

Yurii Vlasov, Univ. of Illinois at Urbana-Champaign, USA

Jonathan Zuegel, Lab for Laser Energetics, Univ. of Rochester, USA

\section{IEEE/Photonics Society}

Ann Catrina Coleman, Univ. of Texas at Dallas, USA

Peter Smowton, Cardiff Univ., UK

Weidong Zhou, Univ. of Texas at Arlington, USA

\section{APS/Division of Laser Science}

Nicholas Bigelow, Univ. of Rochester, USA

Rohit Prasankumar, Los Alamos National Lab, USA

\section{Exhibitor Representatives}

Mark Tolbert, Toptica Photonics Inc., USA

\section{Ex-Officio}

Peter Andersen, Danmarks Tekniske Universitet, Denmark Sterling J. Backus, Kapteyn-Murnane Labs., USA

Seth Bank, Univ. of Texas at Austin, USA

Zhigang Chen, San Francisco State Univ., USA

Kent Choquette, Univ. of Illinois, USA

Ben Eggleton, Univ. of Sydney, Australia

Sasan Fathpour, Univ. of Central Florida, CREOL, USA

Nicusor Iftimia, Physical Sciences Inc., USA

Wilhelm G. Kaenders, Toptica Photonics Inc., Germany

Junichiro Kono, Rice Univ., USA

Michal Lipson, Columbia Univ., USA

Michael M. Mielke, TRUMPF Inc., USA

Roberto Morandotti, INRS-Energie Mat \& Tele Site Varennes, Canada

William Munro, NTT Basic Research Lab, Japan

Nathan Newbury, NIST, USA

Irina Novikova, College of William \& Mary, USA

Valdas Pasiskevicius, Royal Institute of Tech., Sweden

Siddharth Ramachandran, Boston Univ., USA

Jessie Rosenberg, IBM TJ Watson Research Center, USA

Jeff Shapiro, MIT, USA

Dirk H. Sutter, TRUMPF Laser GmbH, Germany

Christian Wetzel, Rensselaer Polytechnic Inst., USA

\section{CLEO Steering ad Hoc Committee on Long Range Planning (LRP)}

Kent Choquette, IEEE/Photonics Society, USA, Chair

Craig Arnold, Princeton Univ., USA

Steve Cundiff, NIST, USA

Wilhelm Kaenders, Toptica Photonics, Germany

Elizabeth A. Rogan, The Optical Society, USA

Mark Tolbert, TOPTICA, USA

\section{CLEO Budget Committee}

Craig Arnold, Princeton Univ., USA

Joe Haus, Univ. of Dayton, USA

Chris Jannuzzi, IEEE Photonics Society, USA

Kate Kirby, American Physical Society, USA

Steve Newton, Keysight Techn., USA

Elizabeth A. Rogan, The Optical Society, USA

Roseanne J. Sension, Univ. of Michigan, USA 


\section{Joint Council on Applications}

Wilhelm G. Kaenders, Toptica Photonics, Germany, Chair Peter Andersen, Danmarks Tekniske Univ., Denmark

Yu Chen, Univ. of Maryland at College Park, USA

Amy Eskilson, Inrad Optics, USA

Peter Fendel, Thorlabs Inc., USA

Paul Ginouves, Coherent Inc., USA

Nicusor Iftimia, Physical Sciences Inc., USA

Jin Kang, John Hopkins Univ., USA

Michael Mielke, Lumentun, USA

Tyler Morgus, Thorlabs Inc., USA

Eric Mottay, Amplitude Systemes, France

Rick Plympton, Optimax Systems, USA

Dirk H. Sutter, TRUMPF Laser GmbH, Germany

Mark Tolbert, Toptica Photonics, USA

Christian Wetzel, Rensselaer Polytechnic Institute, USA

\section{Joint Council on Quantum Electronics}

Nicholas Bigelow, Univ. of Rochester, USA, Chair

Zhigang Chen, San Francisco State University, USA

Demetrios Christodoulides, Univ. of Central Florida, CREOL, USA

Yujie Ding, Lehigh Univ., USA

Ben Eggleton, Univ. of Sydney, Australia

Jacob Khurgin, Purdue Univ., USA

Junichiro Kono, Rice Univ., USA

Alfred Leitenstorfer, Univ. of Konstanz, Germany

Norbert Lutkenhaus, Univ. of Waterloo, Canada

Roberto Morandotti, INRS-Energie Mat \& Tele Site Varennes, Canada

William Munro, NTT Basic Research Lab, Japan

Mikhail Noginov, Norfolk State Univ., USA

Irina Novikova, College of William \& Mary, USA

Rohit Prasankumar, Los Alamos National Laboratory, USA

Jeff Shapiro, MIT, USA

Yurii Vlasov, Univ. of Illinois at Urbana-Champaign, USA 